\title{
Surface polaritons on left-handed cylinders: A complex angular momentum analysis
}

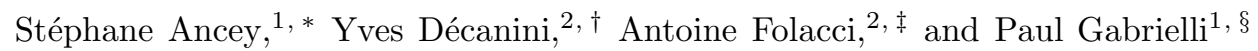 \\ 1 UMR CNRS 6134 SPE, Equipe Ondes et Acoustique, \\ Université de Corse, Faculté des Sciences, Bô̂te Postale 52, 20250 Corte, France \\ ${ }^{2}$ UMR CNRS 6134 SPE, Equipe Physique Semi-Classique (et) de la Matière Condensée, \\ Université de Corse, Faculté des Sciences, Bô̂te Postale 52, 20250 Corte, France
}

(Dated: October 12, 2018)

\begin{abstract}
We consider the scattering of electromagnetic waves by a left-handed cylinder - i.e., by a cylinder fabricated from a left-handed material - in the framework of complex angular momentum techniques. We discuss both the TE and TM theories. We emphasize more particularly the resonant aspects of the problem linked to the existence of surface polaritons. We prove that the long-lived resonant modes can be classified into distinct families, each family being generated by one surface polariton propagating close to the cylinder surface and we physically describe all the surface polaritons by providing, for each one, its dispersion relation and its damping. This can be realized by noting that each surface polariton corresponds to a particular Regge pole of the $S$ matrix of the cylinder. Moreover, for both polarizations, we find that there exists a particular surface polariton which corresponds, in the large-radius limit, to the surface polariton which is supported by the plane interface. There exists also an infinite family of surface polaritons of whispering-gallery type which have no analogs in the plane interface case and which are specific to left-handed materials.
\end{abstract}

PACS numbers: 78.20.Ci, 41.20.Jb, 73.20.Mf, 42.25.Fx

\section{INTRODUCTION}

In an article published in 1967 (see Ref. 1 for the English translation), Veselago speculated upon the alteration of electromagnetic and optical phenomena in hypothetical materials for which the electric permittivity and the magnetic permeability (and therefore the refractive index ${ }^{1.2}$ ) were simultaneously negative in a certain range of frequencies. He predicted more particularly the existence, in such materials, of anomalous effects such as a reversed Doppler shift, reversed Cerenkov radiation, negative radiation pressure, and inverse Snell-Descartes law. Since in such substances the electric field, the magnetic field and the wave vector of a plane wave form a left-handed system, so that the Poynting vector and the wave vector have opposite direction, he referred to them as left-handed media and here we shall use this terminology, even if today other authors prefer to call this kind of materials "negative index media", "double negative media" or "Veselago's media". Four years ago, following insights from Pendry and coworkers ${ }^{3.4 .5}$, Schultz, Smith and colleagues.6.8 have been able to build, for the first time, an artificial left-handed medium by combining arrays of wires and split-ring resonators and to experimentally test "left-handed electromagnetism" in the microwave frequency range. Since then, several other groups have successfully fabricated left-handed media and experimentally studied the alteration of electromagnetic phenomena (see, for a comprehensive list of references, the popular article by Pendry and $\operatorname{Smith}^{9}$ ). Now, the possibility to create left-handed materials working in the optical domain is seriously foreseen and, very recently, negative refraction has been even observed at infrared wavelengths in the context of photonic crys- tal physics $\frac{10}{}$. Of course, the unusual and remarkable properties of left-handed media could revolutionize optoelectronics and communications. Many technological applications are already considered including superlenses, bandpass filters, beam guiders and light-emitting devices. As a consequence, this recent new field of physics has attracted the interest of many researchers and is rapidly evolving and the corresponding scientific literature is exploding.

In this article, we shall focus our attention on a particular problem of left-handed electromagnetism: namely, the scattering of an electromagnetic wave by a "left-handed cylinder" - i.e., by an homogeneous cylinder fabricated from a left-handed material. This problem has been already considered by Kuzmiak and Maradudin ${ }^{11}$ but we shall reexamine it in the framework of complex angular momentum (CAM) techniques ${ }^{12.13}$. In fact, we are above all interested in the resonant aspects of scattering linked to the existence of surface polaritons (SP's) and, by using CAM techniques, we shall be able to provide a theoretical description of SP's propagating close to the left-handed cylinder surface as well as a physical explanation for the excitation of the associated resonant modes.

Let us recall here that SP's are electromagnetic surface waves propagating close to the interface separating two different media with an amplitude that decays in an exponential fashion perpendicularly to the interface and into both media. SP's supported by metal-dielectric or semiconducting-dielectric interfaces have been extensively studied during the last 40 years because of the fundamental role they play in the context of the interaction of electromagnetic radiation with matter but also because of their numerous practical applications in physics, chemistry and biology (for reviews on this subject we refer to 
Refs. 14 15 16). Recently, activity has focused particularly on the role of SP's in photonic crystal physics (see, for example, Refs. 18, 19 20, 21 22 23 24 25 262728 ) as well as on their role in the enhanced transmission of light through periodic arrays of holes in a metal film ${ }^{29}$ (see also Ref. 30 and references therein). Of course, the scientific literature dealing with SP's localized at the interface separating a left-handed medium and a conventional one is much less developed and it is still too early to judge their importance in left-handed electromagnetism. However, it is already obvious that it will not be negligible: indeed, it seems that SP's play a central role in the superlensing phenomenon ${ }^{31.32 .33 .34}$ and in the giant Goos-Hänchen effect recently discovered ${ }^{35}$.

The theory of SP's supported by left-handed media has been studied in Refs. 3637,38 in the case of a plane interface. In that configuration, we can consider that the properties of SP's are now completely known. They have been obtained from rather elementary calculations involving homogeneous and inhomogeneous plane waves. The existence of SP's in the presence of a curved interface has been noted in the article by Kuzmiak and Maradudin 11 previously cited and in an article by Ruppin $^{39}$ dealing with scattering from a sphere. But it should be noted that neither of these two articles provide a clear physical description of SP's. This is mainly due to the fact that, unlike the case for a plane interface, in the case of a curved interface the wave equation cannot be solved in terms of elementary functions. As a consequence, the description of SP's involves transcendental equations which obscures any analysis. Fortunately, it is possible to circumvent these difficulties by using CAM techniques (here, we refer to the Introduction of Ref. 40 for a short bibliography on this topic) in connection with modern aspects of asymptotic $41.42,43.44$. It is exactly what we did in a recent article $\stackrel{40}{\underline{40}}$ dealing with scattering of electromagnetic waves from metallic and semiconducting cylinders - i.e., in the presence of dispersive materials. This allowed us to provide a clear physical explanation for the excitation mechanism of the resonant surface polariton modes (RSPM's) as well as a simple mathematical description of the SP's that generate them. In the present paper, we shall extend this analysis to left-handed cylinders but before we embark on this work we shall briefly discuss the limitations of our approach. We first recall that the CAM method is an asymptotic approach and that formally it is only valid for high frequencies or more precisely when the radius $a$ of the cylinder is large compared to the wavelength $\lambda=2 \pi c / \omega$ of the electromagnetic field. In fact, this limitation must not be taken too seriously. Indeed, in practical applications CAM techniques provide good results even if $\lambda \approx a$. Moreover, in the present problem we cannot assume that $a$ is very large compared to $\lambda$. Indeed, in that case, the wavelength of the electromagnetic field could become comparable to the size $a^{\prime}$ of the unit cell of the left-handed material and the cylinder could not be treated as homogeneous. In other words, the analysis we shall provide in the next sections is formally valid for $a^{\prime} \ll \lambda \leq a$.

Our paper is organized as follows. In Sec. II, we introduce our notations and we construct the $S$ matrix of the system. We consider the TE theory ( $H$ polarization) as well as the TM theory ( $E$ polarization). We then discuss the resonant aspects of our problem for both theories. In Sec. III, by working in the CAM plane, we qualitatively describe the SP's supported by the left-handed cylinder. Then, in Sec. IV, by using CAM techniques, we establish the connection between these SP's and the associated RSPM's. In Sec. V, by using asymptotic techniques, we describe semiclassically the different SP's and we provide analytic expressions for their dispersion relations and their damping. We show more particularly the existence of SP's of whispering-gallery type. Finally, in Sec. VI, we conclude our article by emphasizing the main results of our work.

\section{EXACT $S$ MATRICES AND SCATTERING RESONANCES FOR THE TE AND THE TM THEORIES}

From now on, we consider the scattering of electromagnetic waves by a cylinder with circular cross section and radius $a$ having an effective frequency-dependent permittivity $\epsilon(\omega)$ and an effective frequency-dependent permeability $\mu(\omega)$. Here and in the following, we implicitly assume the time dependence $\exp (-i \omega t)$ for electric and magnetic fields. We consider that the cylinder is embedded in a host medium of infinite extent having the electromagnetic properties of the vacuum. We introduce the usual cylindrical polar coordinate system $(\rho, \theta, z)$. It is chosen so that the cylinder and surrounding medium, respectively, occupy the regions corresponding to the range $0 \leq \rho<a$ (region II) and to the range $\rho>a$ (region I). Furthermore, in order to describe wave propagation we also introduce the wave number

$$
k(\omega)=\frac{\omega}{c}
$$

where $c$ denotes the velocity of light in vacuum, and the refractive index of the cylinder

$$
n(\omega)=\sqrt{\epsilon(\omega) \mu(\omega)} .
$$

As far as the electric permittivity $\epsilon(\omega)$ and the magnetic permeability $\mu(\omega)$ of the cylinder are concerned, we assume they are, respectively, given by

$$
\epsilon(\omega)=1-\frac{\omega_{p}^{2}}{\omega^{2}}
$$

and

$$
\mu(\omega)=1-\frac{F \omega^{2}}{\omega^{2}-\omega_{0}^{2}}=(1-F)\left(\frac{\omega^{2}-\omega_{b}^{2}}{\omega^{2}-\omega_{0}^{2}}\right)
$$

where $0<F<1$ and $\omega_{b}=\omega_{0} / \sqrt{1-F}$. Here we have considered that the cylinder is fabricated from a metamaterial. Of course, the parameters $\omega_{p}, \omega_{0}$ and $F$ depend 
on its structure. But we would like to be as general as possible in our analysis and therefore we do not restrict our study to any particular metamaterial (see, however, the discussion in the last paragraph of our conclusion). As a consequence, we do not attribute any "microscopic" interpretation to the parameters $\omega_{p}, \omega_{0}$ and $F$ in terms of the internal structure of the metamaterial considered. Similarly, we do not precise the frequency range where $\omega_{p}$ and $\omega_{0}$ lie. We only assume that $\omega_{0}<\omega_{b}<\omega_{p}$. We then have $\epsilon(\omega)<0$ in the frequency range $\omega \in] 0, \omega_{p}[$ and $\mu(\omega)<0$ in the frequency range $\omega \in] \omega_{0}, \omega_{b}[$. Thus, the electric permittivity, the magnetic permeability and the refractive index are simultaneously negative in the region $\omega_{0}<\omega<\omega_{b}$. In that region the metamaterial presents left-handed behavior. As far as the numerical aspects of our work are concerned, we shall work with $F=0.4$ and with the reduced frequencies $\omega_{0} a / c=5.52$, $\omega_{b} a / c \approx 7.127$ and $\omega_{p} a / c=11.04$. Even though we restrict ourselves to that particular configuration, the results we shall obtain numerically are in fact very general and they permit us to correctly illustrate the theory.

Here and from now on, we choose to treat our problem in a two-dimensional setting, ignoring the $z$ coordinate. We briefly recall the equations governing the TE theory where the magnetic field $\mathbf{H}$ is parallel to the cylinder axis ( $H$ polarization) and the TM theory where the electric field $\mathbf{E}$ is parallel to the cylinder axis ( $E$ polarization). From Maxwell's equations it is easy to show that the $z$ components of the magnetic and electric fields satisfy the Helmholtz equation

$$
\begin{aligned}
& {\left[\Delta_{\mathbf{x}}+n^{2}(\omega)\left(\frac{\omega}{c}\right)^{2}\right]\left\{\begin{array}{l}
H_{z}^{\mathrm{II}}(\mathbf{x})=0 \\
E_{z}^{\mathrm{II}}(\mathbf{x})=0
\end{array} \text { for } 0 \leq \rho<a\right.} \\
& {\left[\Delta_{\mathbf{x}}+\left(\frac{\omega}{c}\right)^{2}\right]\left\{\begin{array}{l}
H_{z}^{\mathrm{I}}(\mathbf{x})=0 \\
E_{z}^{\mathrm{I}}(\mathbf{x})=0
\end{array} \text { for } \rho>a\right.}
\end{aligned}
$$

with $\mathbf{x}=(\rho, \theta)$ and where the Laplacian $\Delta_{\mathbf{x}}$ is expressed in the polar coordinate system. For the TE theory, from the continuity of the tangential components of the electric and magnetic fields - i.e., of $E_{\theta}$ and $H_{z}$ - at the interface between regions I and II, it can be shown that the $z$ component of the magnetic field satisfies, for $0 \leq \theta<2 \pi$,

$$
\begin{aligned}
& H_{z}^{\mathrm{I}}(\rho=a, \theta)=H_{z}^{\mathrm{II}}(\rho=a, \theta), \\
& \frac{\partial H_{z}^{\mathrm{I}}}{\partial \rho}(\rho=a, \theta)=\frac{1}{\epsilon(\omega)} \frac{\partial H_{z}^{\mathrm{II}}}{\partial \rho}(\rho=a, \theta) .
\end{aligned}
$$

For the TM theory, the continuity of the tangential components of the electric and magnetic fields - i.e., of $E_{z}$ and $H_{\theta}$ - at the interface between regions I and II permits us to show that the $z$ component of the electric field satisfies, for $0 \leq \theta<2 \pi$,

$$
\begin{aligned}
& E_{z}^{\mathrm{I}}(\rho=a, \theta)=E_{z}^{\mathrm{II}}(\rho=a, \theta), \\
& \frac{\partial E_{z}^{\mathrm{I}}}{\partial \rho}(\rho=a, \theta)=\frac{1}{\mu(\omega)} \frac{\partial E_{z}^{\mathrm{II}}}{\partial \rho}(\rho=a, \theta) .
\end{aligned}
$$

We can now construct the $S$ matrix for the cylinder for both polarizations. Because of the cylindrical symmetry of the scatterer, the $S$ matrix is diagonal and its elements $S_{\ell \ell^{\prime}}(\omega)$ are given by $S_{\ell \ell^{\prime}}(\omega)=S_{\ell}(\omega) \delta_{\ell \ell^{\prime}}$. It should be recalled that the $S$ matrix is of fundamental importance because it contains all the information about the scattering process. Its components appear in the Green functions of the problem, in the scattered field when a plane wave excites the cylinder as well as in both the scattering amplitude and the total scattering cross section. For the TE and the TM theories, we shall denote, respectively, by $S_{\ell}^{H}(\omega)$ and $S_{\ell}^{E}(\omega)$ the $S$ matrix diagonal elements. For a given angular momentum index $\ell \in \mathbb{Z}$, the coefficients $S_{\ell}^{H}$ and $S_{\ell}^{E}$ are respectively obtained from the partial wave $\left(H_{z}\right)_{\ell}$ and $\left(E_{z}\right)_{\ell}$ solutions of the following problem ${ }^{45}$ :

(i) $\left(H_{z}\right)_{\ell}$ and $\left(E_{z}\right)_{\ell}$ satisfy the Helmholtz equation (5),

(ii) $\left(H_{z}\right)_{\ell}$ and $\left(E_{z}\right)_{\ell}$, respectively, satisfy the boundary conditions ([6) and (7),

(iii) at large distance, $\left(H_{z}\right)_{\ell}$ and $\left(E_{z}\right)_{\ell}$ respectively present the asymptotic behaviors

$$
\begin{aligned}
\left(H_{z}\right)_{\ell}(\rho, \theta) \underset{\rho \rightarrow+\infty}{\sim} \frac{1}{\sqrt{2 \pi k \rho}}\left(e^{-i(k \rho-\ell \pi / 2-\pi / 4)}\right. \\
\left.+S_{\ell}^{H}(\omega) e^{i(k \rho-\ell \pi / 2-\pi / 4)}\right) e^{i \ell \theta}, \\
\left(E_{z}\right)_{\ell}(\rho, \theta) \underset{\rho \rightarrow+\infty}{\sim} \frac{1}{\sqrt{2 \pi k \rho}}\left(e^{-i(k \rho-\ell \pi / 2-\pi / 4)}\right. \\
\left.+S_{\ell}^{E}(\omega) e^{i(k \rho-\ell \pi / 2-\pi / 4)}\right) e^{i \ell \theta} .
\end{aligned}
$$

Outside the cylinder (region I), the solution of Eq. (5) is expressible in terms of Bessel functions (see Ref. 46) as a linear combination of $J_{\ell}(\omega \rho / c) e^{i \ell \theta}$ and $H_{\ell}^{(1)}(\omega \rho / c) e^{i \ell \theta}$. Inside the cylinder (region II), it is proportional to $J_{\ell}(n(\omega) \omega \rho / c) e^{i \ell \theta}$. As a consequence, the partial waves $\left(H_{z}\right)_{\ell}$ and $\left(E_{z}\right)_{\ell}$ solutions of (i) and (ii) can be obtained exactly. Then, by using the standard asymptotic behavior of Hankel functions $H_{\ell}^{(1)}(x)$ and $H_{\ell}^{(2)}(x)$ for $x \rightarrow \infty$ (see Ref. 46), we find from (iii) the expressions of the diagonal elements $S_{\ell}^{H}$ and $S_{\ell}^{E}$ of the $S$ matrix for the TE and the TM theories. We have

$$
S_{\ell}^{H}(\omega)=1-2 \frac{C_{\ell}^{H}(\omega)}{D_{\ell}^{H}(\omega)} \text { and } S_{\ell}^{E}(\omega)=1-2 \frac{C_{\ell}^{E}(\omega)}{D_{\ell}^{E}(\omega)}
$$

where $C_{\ell}^{H}(\omega), D_{\ell}^{H}(\omega), C_{\ell}^{E}(\omega)$ and $D_{\ell}^{E}(\omega)$ are $2 \times 2$ determinants which are explicitly given by

$$
\begin{aligned}
C_{\ell}^{H}(\omega)= & \sqrt{\epsilon(\omega) / \mu(\omega)} J_{\ell}^{\prime}(\omega a / c) J_{\ell}(n(\omega) \omega a / c) \\
& -J_{\ell}(\omega a / c) J_{\ell}^{\prime}(n(\omega) \omega a / c), \\
D_{\ell}^{H}(\omega)= & \sqrt{\epsilon(\omega) / \mu(\omega)} H_{\ell}^{(1)^{\prime}}(\omega a / c) J_{\ell}(n(\omega) \omega a / c) \\
& -H_{\ell}^{(1)}(\omega a / c) J_{\ell}^{\prime}(n(\omega) \omega a / c),
\end{aligned}
$$



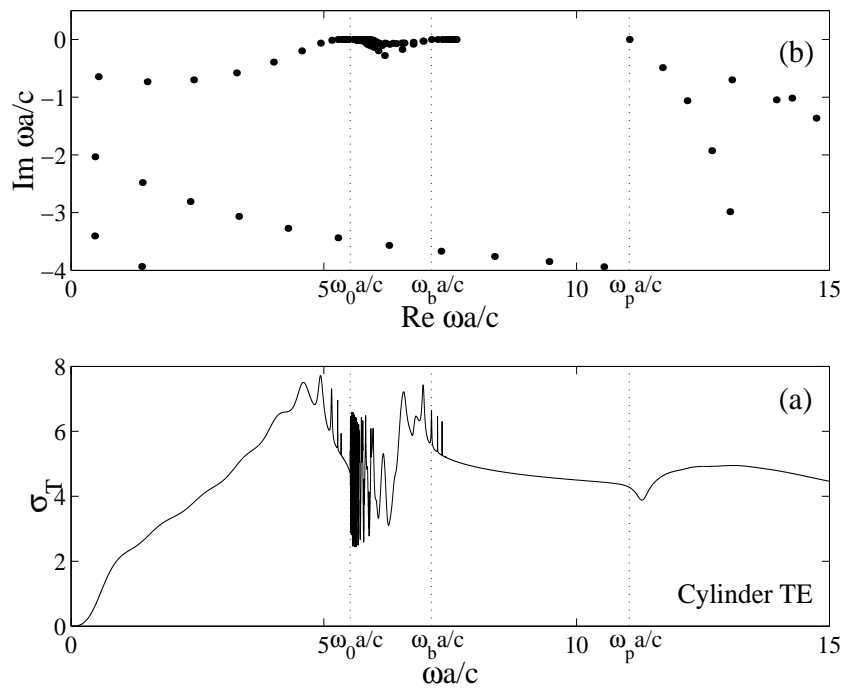

FIG. 1: TE theory. a) Total cross section $\sigma_{T}^{H}$. b) Scattering resonances in the complex $\omega a / c$ plane.
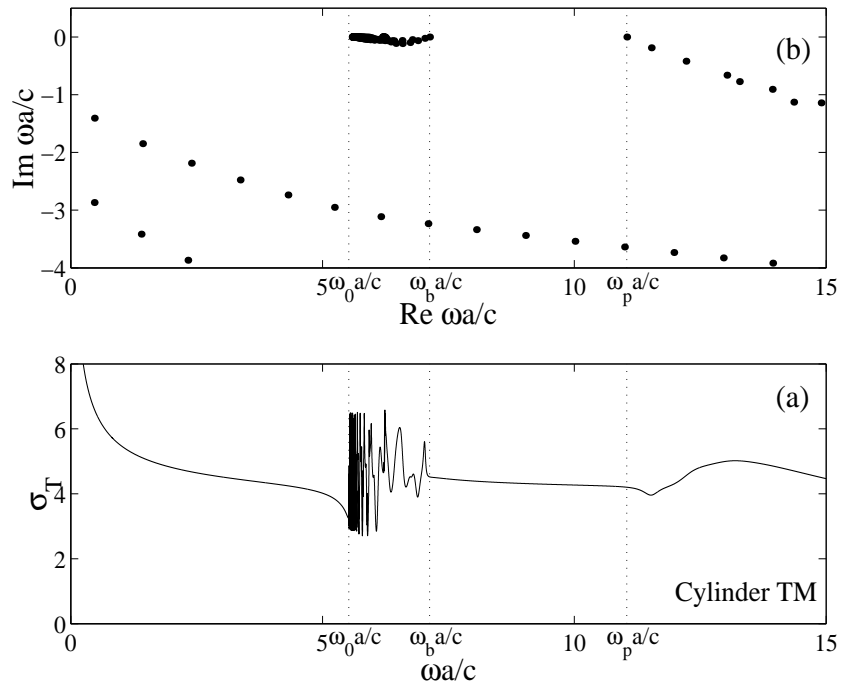

FIG. 2: TM theory. a) Total cross section $\sigma_{T}^{E}$. b) Scattering resonances in the complex $\omega a / c$ plane.

and

$$
\begin{aligned}
C_{\ell}^{E}(\omega)= & \sqrt{\mu(\omega) / \epsilon(\omega)} J_{\ell}^{\prime}(\omega a / c) J_{\ell}(n(\omega) \omega a / c) \\
& -J_{\ell}(\omega a / c) J_{\ell}^{\prime}(n(\omega) \omega a / c), \\
D_{\ell}^{E}(\omega)= & \sqrt{\mu(\omega) / \epsilon(\omega)} H_{\ell}^{(1)^{\prime}}(\omega a / c) J_{\ell}(n(\omega) \omega a / c) \\
& -H_{\ell}^{(1)}(\omega a / c) J_{\ell}^{\prime}(n(\omega) \omega a / c) .
\end{aligned}
$$

For both polarizations, the unitarity of the $S$ matrix ${ }^{12}$, which expresses the energy conservation, and the reciprocity property ${ }^{12}$, which is associated with time-reversal invariance, can be easily verified by using elementary properties of Bessel functions.

As far as the scattering amplitude $f(\omega, \theta)$ and the total scattering cross section per unit length of the cylinder
$\sigma_{T}(\omega)$ are concerned, they are, respectively, given by

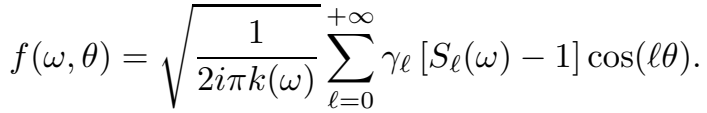

where $\gamma_{\ell}$ is the Neumann factor $\left(\gamma_{0}=1\right.$ and for $\ell \neq 0$, $\gamma_{\ell}=2$ ) and

$$
\sigma_{T}(\omega)=\sqrt{\frac{8 \pi}{k(\omega)}} \operatorname{Im}\left(e^{-i \pi / 4} f(\omega, \theta=0)\right) .
$$

It should be noted that the definition (12) assumes the unitarity of the $S$ matrix. It is obtained by using the optical theorem ${ }^{12}$. From the expressions (8)-(10) of the $S$ matrices and by using expressions (11) and (12), we obtain the total cross sections $\sigma_{T}^{H}(\omega)$ and $\sigma_{T}^{E}(\omega)$ for the TE and TM theories. We have

$$
\sigma_{T}^{H}(\omega)=\frac{4}{k(\omega)} \sum_{\ell=0}^{+\infty} \gamma_{\ell}\left|\frac{C_{\ell}^{H}(\omega)}{D_{\ell}^{H}(\omega)}\right|^{2}
$$

and

$$
\sigma_{T}^{E}(\omega)=\frac{4}{k(\omega)} \sum_{\ell=0}^{+\infty} \gamma_{\ell}\left|\frac{C_{\ell}^{E}(\omega)}{D_{\ell}^{E}(\omega)}\right|^{2}
$$

Here, it should be noted that our expressions for the total cross sections $\sigma_{T}^{H}(\omega)$ and $\sigma_{T}^{E}(\omega)$ are different from the expressions given by Kuzmiak and Maradudin ${ }^{11}$. It seems to us that these authors have incorrectly applied the boundary conditions for the electric and magnetic fields at the cylinder surface.

In Figs. 11a and 2a, we display the total cross sections for the TE and the TM theories. They are both plotted as functions of the reduced frequency $w a / c$. In the two figures, rapid variations of sharp characteristic shapes can be observed. For the $H$ polarization, this strongly fluctuating behavior is localized within and slightly around the frequency range where the cylinder presents left-handed behavior, while for the $E$ polarization, it is totally localized within that frequency range. For both polarizations, such strongly fluctuating behavior is due to the scattering resonances associated with the long-lived resonant modes of the cylinder - i.e., the long-lived resonant states of the photon-cylinder system. These resonances are the poles of the $S$ matrix lying in the fourth quadrant of the complex $\omega$ plane near the real $\omega$ axis. Resonances are determined by solving

$$
D_{\ell}^{H}(\omega)=0 \quad \text { for } \quad \ell \in \mathbb{N}
$$

for the TE theory and

$$
D_{\ell}^{E}(\omega)=0 \quad \text { for } \quad \ell \in \mathbb{N}
$$

for the TM theory. In Figs. 10 and 2], resonances are exhibited for both theories. For certain frequencies, we can clearly observe a one-to-one correspondence between 
the peaks of $\sigma_{T}(\omega)$ and the resonances near the real $\omega a / c$ axis but in general the situation seems very confused. This is due to the profusion of long-lived resonant modes in and around the frequency range where the cylinder presents left-handed behavior. Furthermore, by zooming in on the distribution of resonances in regions close to the real axis of the complex $\omega$ plane, we have also observed accumulations of resonances for large values of $\ell$ :

- For the TE theory, there exists an accumulation of resonances which converges to the limiting frequency $\omega_{s}$ satisfying

$$
\epsilon\left(\omega_{s}\right)+1=0
$$

and given by

$$
\omega_{s}=\frac{\omega_{p}}{\sqrt{2}}
$$

We have for the corresponding numerical reduced frequency $\omega_{s} a / c \approx 7.806$.

- For the TM theory, there exists an accumulation of resonances at the limiting frequency $\omega_{f}$ satisfying

$$
\mu\left(\omega_{f}\right)+1=0
$$

and given by

$$
\omega_{f}=\omega_{0} \sqrt{\frac{2}{2-F}} .
$$

We have for the corresponding numerical reduced frequency $\omega_{f} a / c \approx 6.172$.

- For both theories, there exists an accumulation of resonances at the pole $\omega_{0}$ of $\mu(\omega)$ and which corresponds more precisely to

$$
\mu\left(\omega_{0}\right)=-\infty .
$$

In summary, as far as the spectrum of resonances is concerned, the left-handed cylinder is a physical system much richer than the metallic or the semiconducting cylinder (see Figs. 1 and 2 of Ref. 40 and the discussion at the end of Sec. II of that reference) and this is certainly very interesting for practical applications of left-handed electromagnetism. With this aim in view, it is necessary to understand the resonance spectrum of the left-handed cylinder from a physical point of view - i.e., to decode the underlying physics. That is what we shall do in the next sections. More precisely, we shall prove that this resonance spectrum is generated by SP's orbiting around the cylinder and we shall provide a numerical and a theoretical description of these surface waves.

It should be noted that the spectrum of resonances lies beyond the frequency range where $n(\omega)<0$ (long-wave and short-wave scattering). The corresponding resonant modes are associated with bulk polaritons. Their complex resonances appear in Figs. 1b and 2b. Their imaginary parts are much larger in modulus than those associated with the resonant modes generated by SP's. As

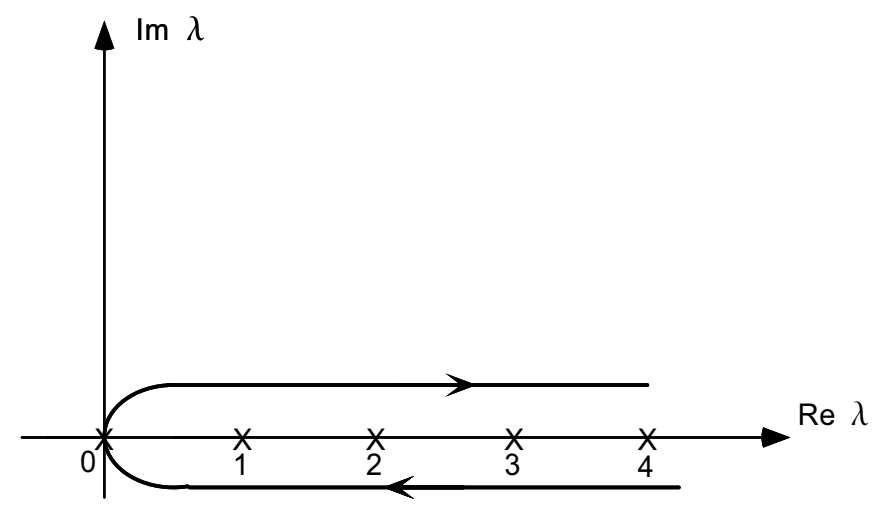

FIG. 3: The Watson integration contour.

a consequence, they have a shorter lifetime, they do not play a significant role in the scattering process (see Fig.1a and Fig.2a beyond the frequency range where $n(\omega)<0$ ) and so they are much less interesting with in mind practical applications. For all these reasons, we focus our interest only on the resonant modes associated with surface polaritons.

\section{REGGE POLES AND SURFACE POLARITONS}

Using the CAM method, we shall first provide a physical picture of the scattering of electromagnetic waves by the left-handed cylinder in term of diffraction by surface waves. By means of a Watson transformation 47 applied to the scattering amplitude (11), we can write

$$
f(\omega, \theta)=\sqrt{\frac{i}{2 \pi k(\omega)}} \mathcal{P} \int_{\mathcal{C}} \frac{\left(S_{\lambda}(\omega)-1\right)}{\sin \pi \lambda} \cos [\lambda(\pi-\theta)] d \lambda .
$$

Here, in order to simplify the notation, we have not specified the polarization. In Eq. (22), $\mathcal{C}$ is the integration contour in the complex $\lambda$ plane (CAM plane) illustrated in Fig. [3] and which encircles the real axis in the clockwise sense. $\mathcal{P}$ which stands for Cauchy's principal value at the origin is used in order to reproduce the Neumann factor. The Watson transformation has permitted us to replace the ordinary angular momentum $\ell$ by the complex angular momentum $\lambda . S_{\lambda}(\omega)$ is now an analytic extension of $S_{\ell}(\omega)$ into the complex $\lambda$ plane which is regular in the vicinity of the positive real $\lambda$ axis. Using Cauchy's theorem and by noting that inside the contour $\mathcal{C}$ the only singularities of the integrand in (22) are the integers, we can easily recover (11) from (22).

We can then deform the path of integration in (22) taking into account the possible singularities. The only singularities that are encountered are the poles of the $\mathrm{S}$ matrix lying in the CAM plane. They are known as Regge poles ${ }^{12,13}$ and are determined by solving

$$
D_{\lambda}^{H}(\omega)=0 \text { for } \omega>0
$$



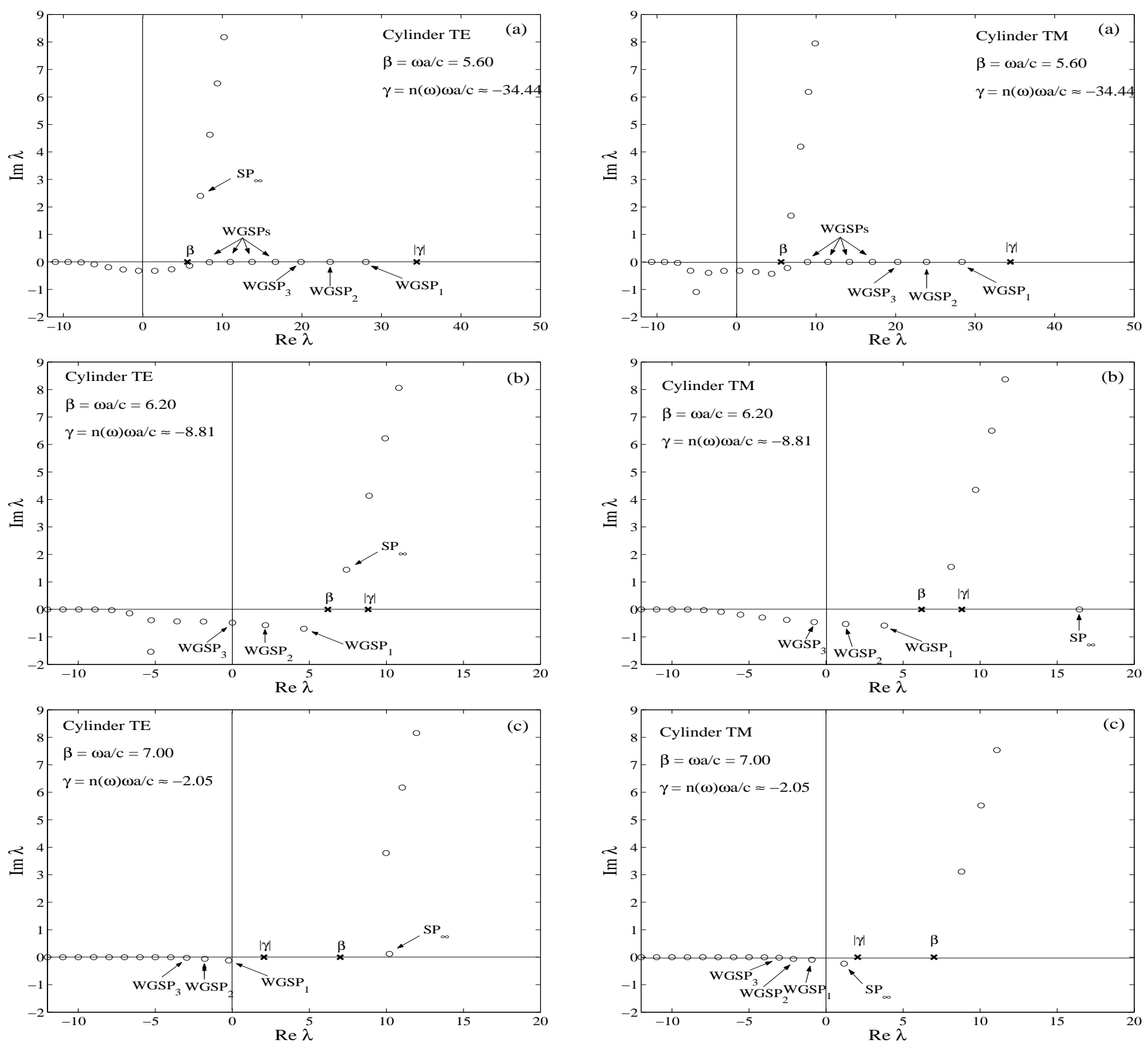

FIG. 4: Regge poles in the CAM plane for the TE theory. a) The distribution corresponds to $\omega a / c=5.6$. b) The distribution corresponds to $\omega a / c=6.2$. c) The distribution corresponds to $\omega a / c=7.0$.

for the TE theory and

$$
D_{\lambda}^{E}(\omega)=0 \quad \text { for } \quad \omega>0
$$

for the TM theory. Figures 4 and 15 exhibit the distribution of Regge poles for both theories for three different reduced frequencies lying in the frequency region where $n(\omega)<0$. At first sight, these Regge pole distributions are more complicated than the distributions associated with the metallic and the semiconducting cylinders studied in Ref. 40. However, we have identified and indicated some particular Regge poles which, as we shall see be-

FIG. 5: Regge poles in the CAM plane for the TM theory. a) The distribution corresponds to $\omega a / c=5.6$. b) The distribution corresponds to $\omega a / c=6.2$. c) The distribution corresponds to $\omega a / c=7.0$.

low, are associated with surface waves orbiting around the cylinder and which explain its resonant behavior. For both polarizations:

- One of these Regge poles is associated with the $\mathrm{SP}$ denoted $\mathrm{SP}_{\infty}$ which, as we shall show in Sec. V, corresponds, in the large-radius limit (i.e., for $a \rightarrow \infty$ ), to a SP which is supported by the plane interface and which has been theoretically described in Refs. 36 37.38.

- The other Regge poles are associated with an infinite family of SP's of whispering-gallery type denoted by $\mathrm{WGSP}_{n}$ with $n \in \mathbb{N}$ and which have no analogs in the 
plane interface case (see Sec. V).

For the TE theory, the Regge pole $\lambda_{\mathrm{SP}_{\infty}}$ lies in the first quadrant of the CAM plane while for the TM theory it lies in the fourth quadrant of that plane. For both theories, the Regge poles $\lambda_{\mathrm{WGSP}_{n}}$ lie in the fourth quadrant of the CAM plane but as $\omega \rightarrow \omega_{b}$ they migrate to the third quadrant of that plane where they do not play any role.

By Cauchy's theorem we can now extract from (22) the contribution of a residue series over Regge poles. For each Regge pole $\lambda_{\mathrm{SP}}(\omega)$ (here $\mathrm{SP}$ stands for $\mathrm{SP}_{\infty}$ as well for $\mathrm{WGSP}_{n}$ ), we capture an associated contribution given by

$$
f_{\mathrm{SP}}(\omega, \theta)=\sqrt{\frac{2 \pi}{i k(\omega)}} \frac{r_{\mathrm{SP}}(\omega)}{\sin \left[\pi \lambda_{\mathrm{SP}}(\omega)\right]} \cos \left[\lambda_{\mathrm{SP}}(\omega)(\pi-\theta)\right]
$$

where $r_{\mathrm{SP}}(\omega)=$ residue $\left(S_{\lambda}(\omega)\right)_{\lambda=\lambda_{\mathrm{SP}}(\omega)}$.

By using

$$
\frac{1}{\sin \pi \lambda}=-2 i \sum_{m=0}^{+\infty} e^{+i \pi(2 m+1) \lambda}
$$

which is true if $\operatorname{Im} \lambda>0$, we can write

$$
\begin{aligned}
f_{\mathrm{SP}}(\omega, \theta) & =-\sqrt{\frac{2 i \pi}{k(\omega)}} r_{\mathrm{SP}}(\omega) \\
\times & \sum_{m=0}^{+\infty}\left(e^{+i \lambda_{\mathrm{SP}}(\omega)(\theta+2 m \pi)}+\right. \\
& \left.e^{+i \lambda_{\mathrm{SP}}(\omega)(2 \pi-\theta+2 m \pi)}\right)
\end{aligned}
$$

when the Regge pole $\lambda_{\text {SP }}$ lies in the first quadrant of the CAM plane. Expression (26) is therefore associated with the surface wave $\mathrm{SP}_{\infty}$ of the TE theory. By using

$$
\frac{1}{\sin \pi \lambda}=2 i \sum_{m=0}^{+\infty} e^{-i \pi(2 m+1) \lambda}
$$

which is true if $\operatorname{Im} \lambda<0$, we can write

$$
\begin{aligned}
& f_{\mathrm{SP}}(\omega, \theta)=+\sqrt{\frac{2 i \pi}{k(\omega)}} r_{\mathrm{SP}}(\omega) \\
& \times \sum_{m=0}^{+\infty}\left(e^{-i \lambda_{\mathrm{SP}}(\omega)(\theta+2 m \pi)}+\right. \\
&\left.e^{-i \lambda_{\mathrm{SP}}(\omega)(2 \pi-\theta+2 m \pi)}\right)
\end{aligned}
$$

when the Regge pole $\lambda_{\mathrm{SP}}$ lies in the fourth quadrant of the CAM plane. Expression (27) is therefore associated with the surface waves $\mathrm{WGSP}_{n}$ with $n \in \mathbb{N}$ of the TE theory as well as with the surface waves $\mathrm{SP}_{\infty}$ and $\mathrm{WGSP}_{n}$ with $n \in \mathbb{N}$ of the TM theory.

In Eqs. (26) and (27), exponential terms correspond to diffractive contributions. This clearly appears by taking into account the time dependence $\exp (-i \omega t)$. The physical interpretations slightly differ according to the position of the Regge pole $\lambda_{\mathrm{SP}}$ in the complex $\lambda$ plane:

- When the Regge pole $\lambda_{\mathrm{SP}}(\omega)$ lies in the first quadrant of the CAM plane - i.e., when we can use Eq. 26) - the term $\exp \left[i \lambda_{\mathrm{SP}}(\omega)(\theta)\right]$ (resp. $\left.\exp \left[i \lambda_{\mathrm{SP}}(\omega)(2 \pi-\theta)\right]\right)$ describes the SP propagating counterclockwise (resp. clockwise) around the cylinder and $\operatorname{Re} \lambda_{\mathrm{SP}}(\omega)$ represents its azimuthal propagation constant while $\operatorname{Im} \lambda_{\mathrm{SP}}(\omega)$ is its damping constant. The corresponding exponential decay reads $\exp \left[-\operatorname{Im} \lambda_{\mathrm{SP}}(\omega) \theta\right]$ (resp. $\left.\exp \left[-\operatorname{Im} \lambda_{\mathrm{SP}}(\omega)(2 \pi-\theta)\right]\right)$.

- When the Regge pole $\lambda_{\mathrm{SP}}(\omega)$ lies in the fourth quadrant of the CAM plane - i.e., when we can use Eq. (27) - the term $\exp \left[-i \lambda_{\mathrm{SP}}(\omega)(\theta)\right]$ (resp. $\left.\exp \left[-i \lambda_{\mathrm{SP}}(\omega)(2 \pi-\theta)\right]\right)$ describes the SP propagating clockwise (resp. counterclockwise) around the cylinder and $\operatorname{Re} \lambda_{\mathrm{SP}}(\omega)$ represents its azimuthal propagation constant while $-\operatorname{Im} \lambda_{\mathrm{SP}}(\omega)$ is its damping constant. The corresponding exponential decay reads $\exp \left[+\operatorname{Im} \lambda_{\mathrm{SP}}(\omega) \theta\right]$ (resp. $\left.\exp \left[+\operatorname{Im} \lambda_{\mathrm{SP}}(\omega)(2 \pi-\theta)\right]\right)$.

Finally, in both cases, the sum over $m$ in (26) and (27) takes into account the multiple circumnavigations of the surface wave around the cylinder as well as the associated radiation damping.

From the previous discussion, it is important to keep in mind that the function $\operatorname{Re} \lambda_{\mathrm{SP}}(\omega)$ provides the dispersion relation for the SP associated with the Regge pole $\lambda_{\mathrm{SP}}$ and that the phase velocity $v_{p}$ and the group velocity $v_{g}$ of that SP are therefore given by

$$
v_{p}=\frac{a \omega}{\operatorname{Re} \lambda_{\mathrm{SP}}(\omega)} \quad \text { and } \quad v_{g}=\frac{d a \omega}{d \operatorname{Re} \lambda_{\mathrm{SP}}(\omega)} .
$$

Here we have taken into account the fact that the SP is supported by the cylinder surface at $\rho=a$ and therefore that its wave number is given by

$$
k_{\mathrm{SP}}(\omega)=\frac{\operatorname{Re} \lambda_{\mathrm{SP}}(\omega)}{a} .
$$

Moreover, it is also important to note that the Regge poles $\lambda_{\mathrm{SP}_{\infty}}$ and $\lambda_{\mathrm{WGSP}_{n}}$ with $n \in \mathbb{N}$ are always close to the real axis in the complex $\lambda$ plane. As a consequence, they all correspond to SP's which are slightly attenuated during their propagation and which contribute significantly to the scattering process and to the resonance excitation mechanism.

As $\omega$ varies, a given Regge pole $\lambda_{\mathrm{SP}}(\omega)$ describes a curve in the CAM plane. Such a curve is called a Regge trajectory $\underline{\underline{12}}$. From a physical point of view, the Regge trajectory corresponding to $\lambda_{\mathrm{SP}}(\omega)$ provides the dispersion relation as well as the damping of the surface wave associated with this pole. In Figs. 6.9] we have displayed the Regge trajectories of some SP's for the TE and TM theories. They have been obtained by solving numerically Eqs. (23) and (24). We can observe some interesting features:

- The dispersion curve for the surface wave $\mathrm{SP}_{\infty}$ of the TE theory is a positive and monotonically increasing function of $\omega$. As a consequence, the associated group 

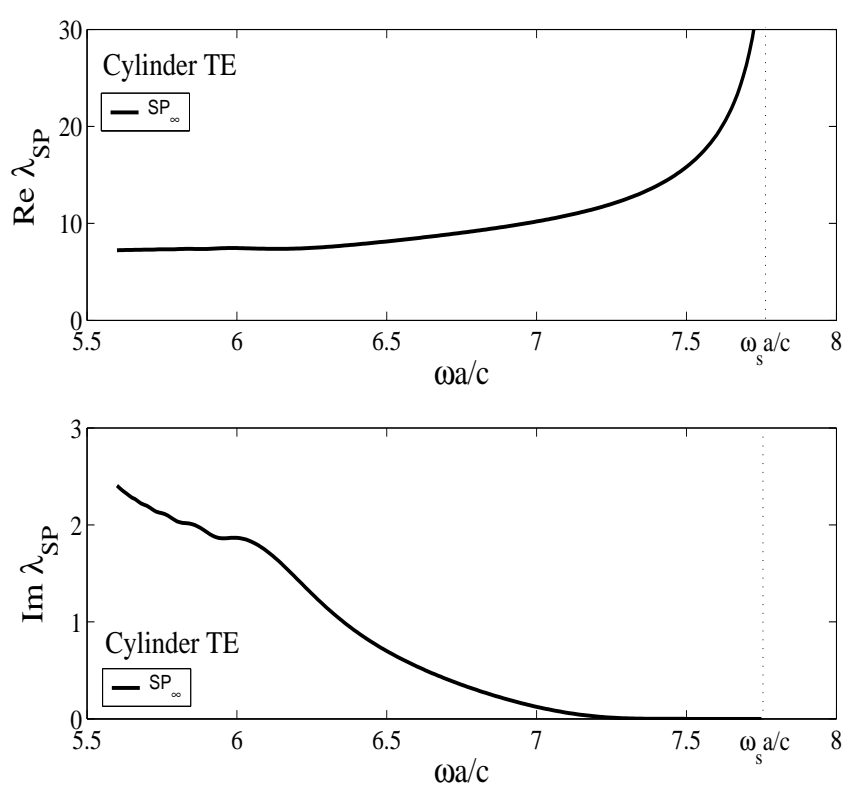

FIG. 6: Regge trajectory for the Regge pole associated with $\mathrm{SP}_{\infty}$ (TE theory).
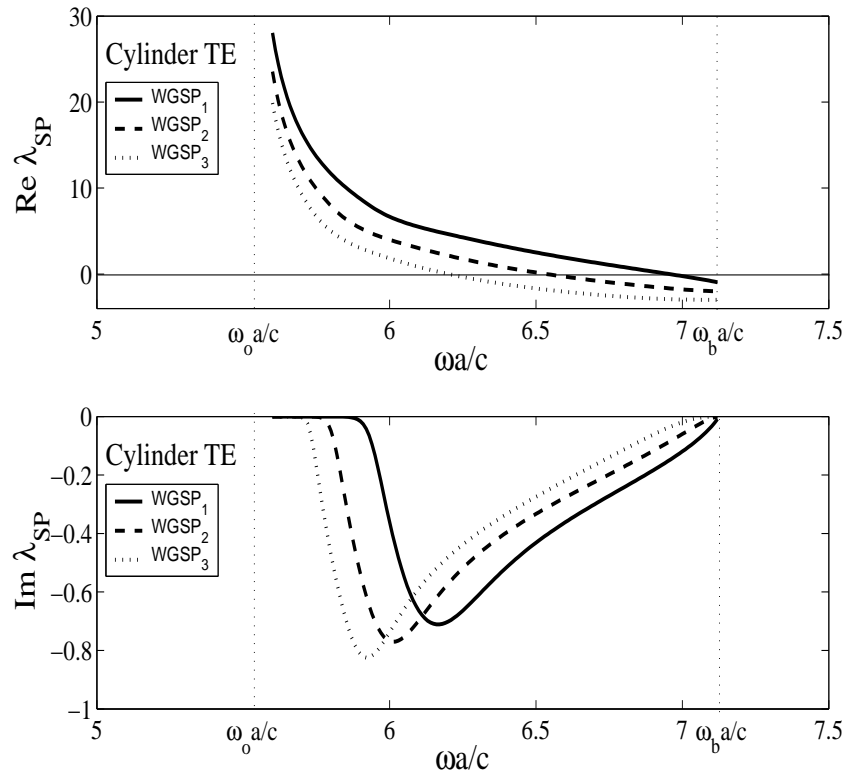

FIG. 7: Regge trajectories for the Regge poles associated with the first three whispering-gallery SP's (TE theory).

and phase velocities given by Eq. (28) are both positive and $\mathrm{SP}_{\infty}$ has an ordinary behavior. It should be also noted that this SP exists in the frequency range $\omega \in] 0, \omega_{s}[$ and therefore in the range $] \omega_{0}, \omega_{b}[$ where the refraction index is negative but also outside this range. However, for low values of $\omega$, its damping becomes very high and thus this surface wave has a negligible role in the scattering process and in the resonance excitation mechanism. Furthermore, it should be noted that as $\omega \rightarrow \omega_{s}$ the dispersion curve increases indefinitely. In the next section, this result will permit us to explain the accumulation of
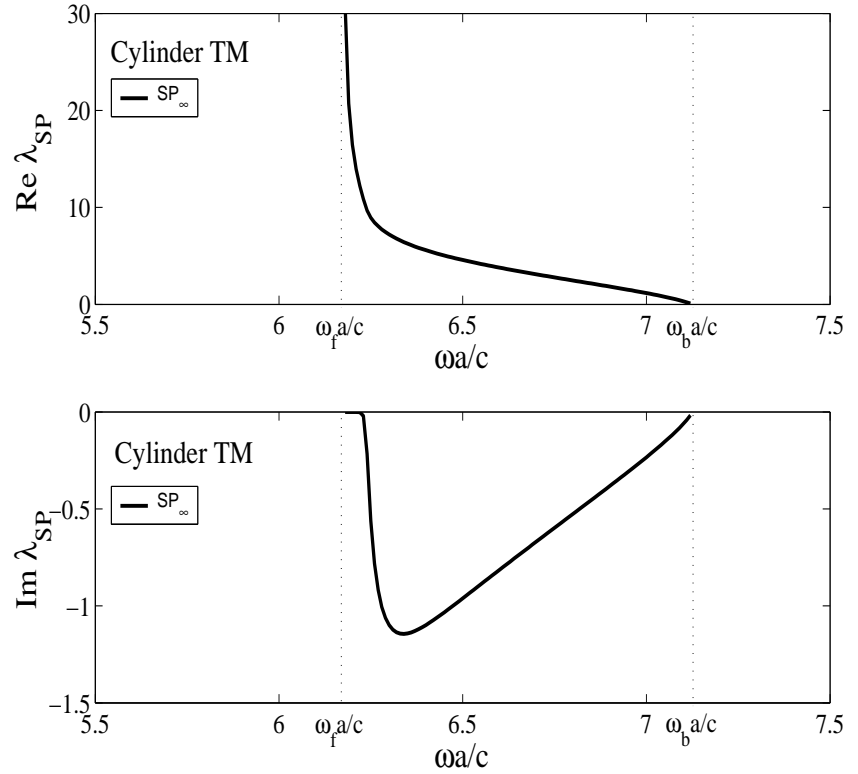

FIG. 8: Regge trajectory for the Regge pole associated with $\mathrm{SP}_{\infty}$ (TM theory).
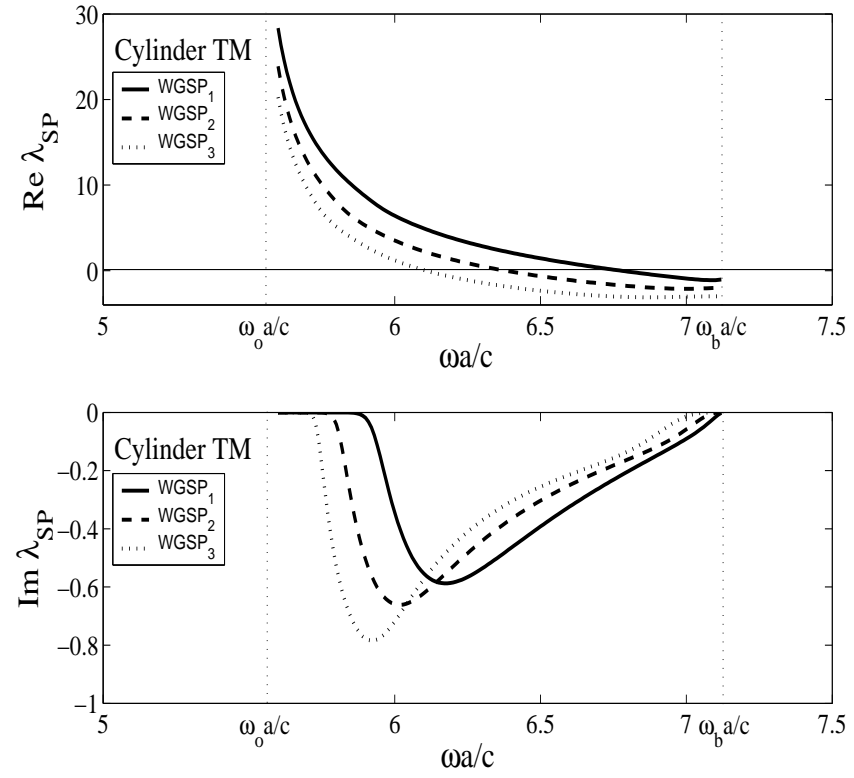

FIG. 9: Regge trajectories for the Regge poles associated with the first three whispering-gallery SP's (TM theory).

resonances which converges to the limiting frequency $\omega_{s}$ for the TE theory.

- The dispersion curve for the surface wave $\mathrm{SP}_{\infty}$ of the TM theory is a positive and monotonically decreasing function of $\omega$. As a consequence, the associated phase velocity is positive while the group velocity is negative (see Eq. (28)). $\mathrm{SP}_{\infty}$ has a "left-handed behavior". It should be also noted that this SP only exists in the frequency range $] \omega_{f}, \omega_{b}[$ which is included in the frequency range $] \omega_{0}, \omega_{b}[$ where the refraction index is negative, that its damping is always weak and thus that this 
surface wave always plays a significant role in the scattering process and in the resonance excitation mechanism. Finally, it should be noted that as $\omega \rightarrow \omega_{f}$ the dispersion curve increases indefinitely. In the next section, this result will permit us to explain the accumulation of resonances which converges to the limiting frequency $\omega_{f}$ for the TM theory.

- As far as the surface waves $\mathrm{WGSP}_{n}$ with $n \in \mathbb{N}$ of the TE and TM theories are concerned, it seems at first sight they present a behavior which is rather independent of the polarization. It should be also noted that the real part of a given Regge pole $\lambda_{\mathrm{WGSP}_{n}}$ vanishes for a frequency in the frequency range $] \omega_{0}, \omega_{b}[$ and becomes negative. The Regge pole then lies in the third quadrant of the CAM plane and is not taken into account by the theory previously developed. So we can consider that the surface waves $\mathrm{WGSP}_{n}$ with $n \in \mathbb{N}$ only exist in a sub-domain of the frequency range $] \omega_{0}, \omega_{b}[$ where the refraction index is negative. The dispersion relations of all these surface waves are positive but monotonically decreasing functions. Their group velocities are always negative while their phase velocities are positive (see Eq. (28)). All these SP's thus have a "left-handed behavior". Furthermore, because the dampings of these surface waves are always weak, they all play a significant role in the scattering process and in the resonance excitation mechanism. Finally, it should be noted that as $\omega \rightarrow \omega_{0}$, the dispersion curves increase indefinitely. In the next section, this result will permit us to explain the accumulation of resonances which converges to the limiting frequency $\omega_{0}$ for the TE and TM theories.

\section{FROM SURFACE POLARITONS TO RESONANCES}

From now on, we shall focus our attention on the physical interpretation of the long-lived resonant modes whose excitation frequencies are localized within or slightly around the frequency range $\omega_{0}<\omega<\omega_{b}$ where the cylinder presents left-handed behavior. In this section, we shall prove that they are generated by the SP's described in Sec. III and for this reason we have called them RSPM's.

We first consider that the solutions of (15) or (16) are denoted by $\omega_{\ell p}=\omega_{\ell p}^{(0)}-i \Gamma_{\ell p} / 2$ where $\omega_{\ell p}^{(0)}>0$ and $\Gamma_{\ell p}>0$, the index $p$ permitting us to distinguish between the different roots of (15) or (16) for a given $\ell$. We also assume that in the immediate neighborhood of the resonance $\omega_{\ell p}, S_{\ell}^{H}(\omega)$ or $S_{\ell}^{E}(\omega)$ and therefore the corresponding scattering amplitudes obtained from (11) present a Breit-Wigner type behavior - i.e., are proportional to

$$
\frac{\Gamma_{\ell p} / 2}{\omega-\omega_{\ell p}^{(0)}+i \Gamma_{\ell p} / 2} .
$$

The resonant behavior of the cylinder (or more precisely of the cylinder-photon system) can then be understood

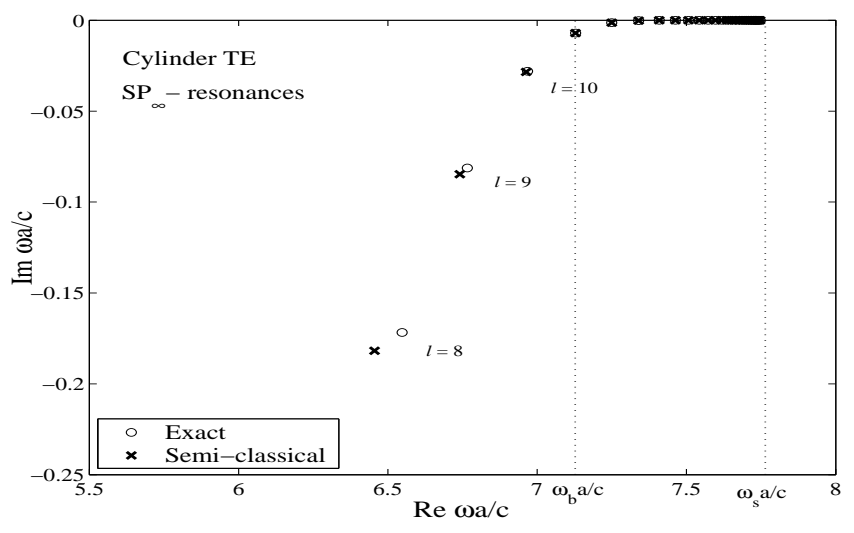

FIG. 10: Resonances generated by $\mathrm{SP}_{\infty}$ (TE theory).
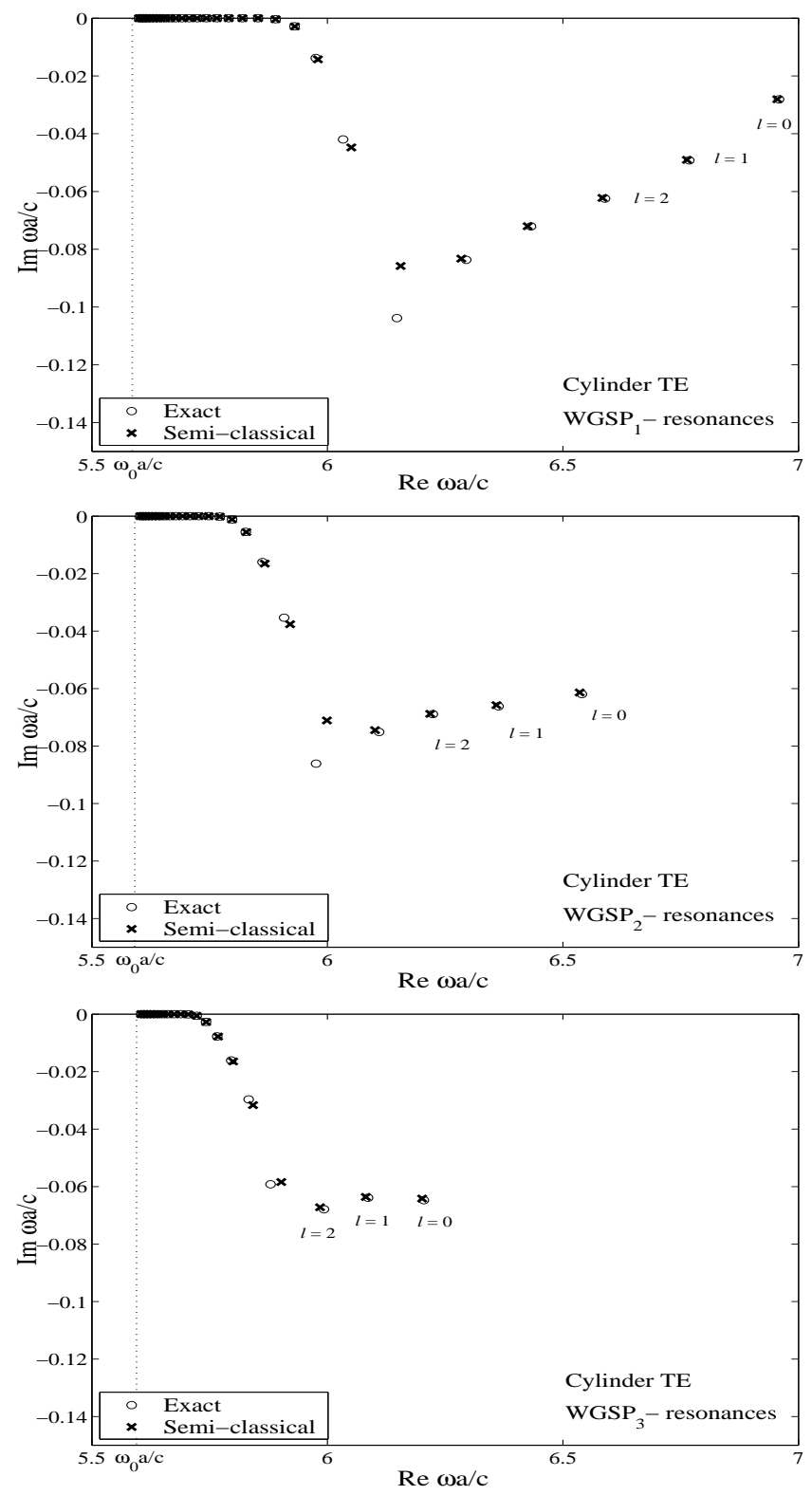

FIG. 11: Resonances generated by the first three whisperinggallery SP's (TE theory). 


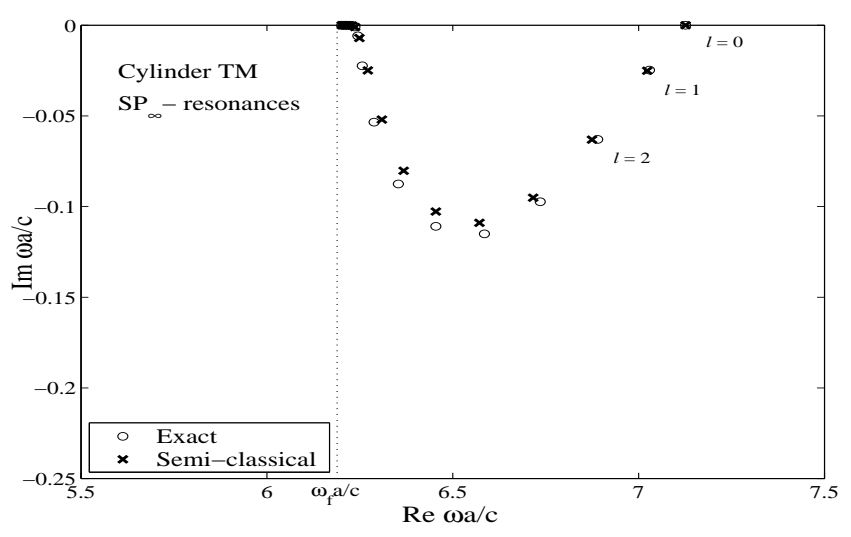

FIG. 12: Resonances generated by $\mathrm{SP}_{\infty}$ (TM theory).
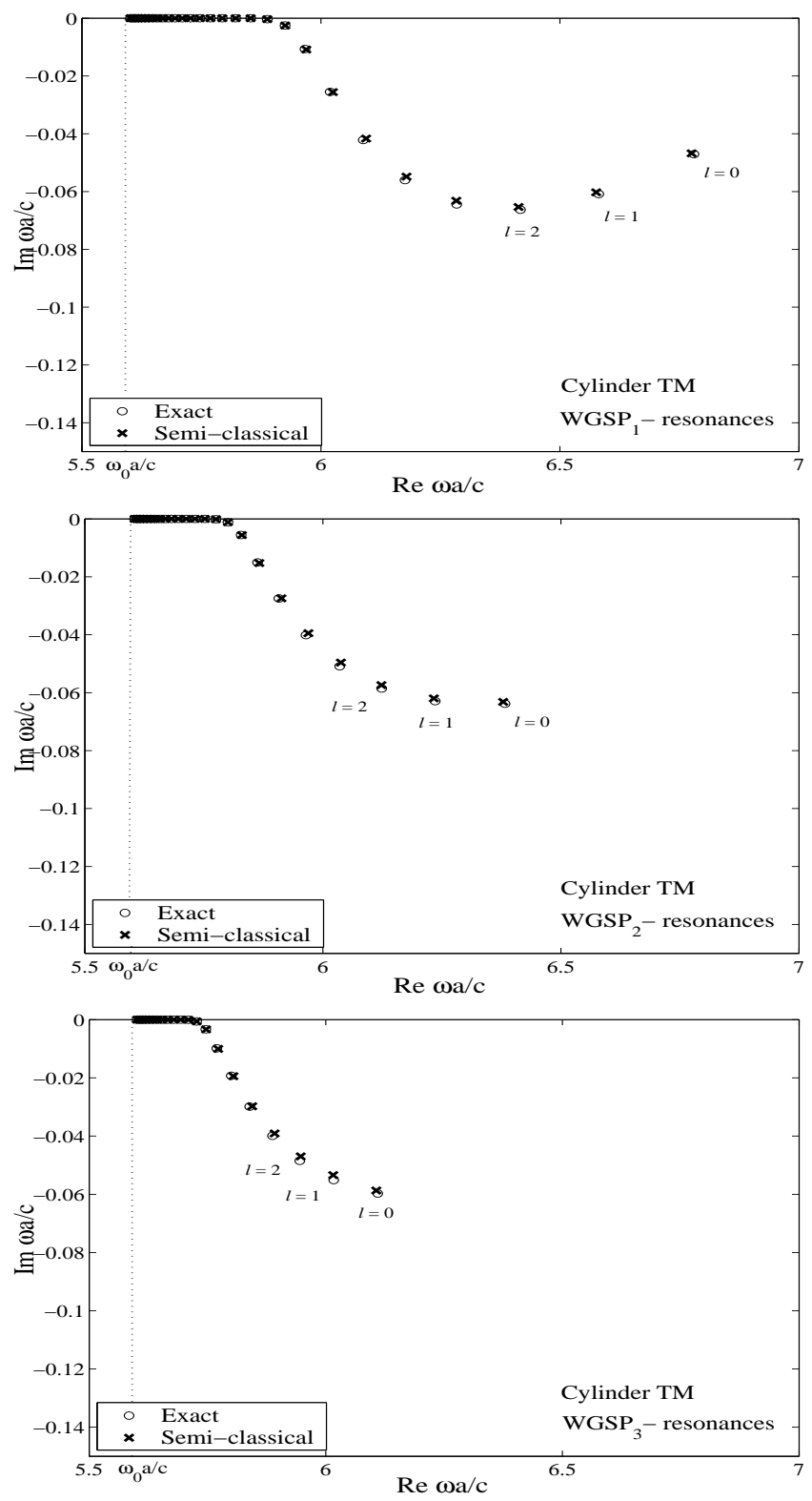

FIG. 13: Resonances generated by the first three whisperinggallery SP's (TM theory). in terms of SP Regge trajectories. Let us consider a given SP (we do not precise its polarization) described by the Regge pole denoted $\lambda_{\mathrm{SP}}(\omega)$. When the quantity $\operatorname{Re} \lambda_{\mathrm{SP}}(\omega)$ coincides with an integer, a resonance occurs which is produced by a constructive interference between the different components of the surface wave, each component corresponding to a different number of circumnavigations of the cylinder (see Eqs. (26) and (27). Resonance excitation frequencies $\omega_{\ell S P}^{(0)}$ associated with that SP are therefore obtained from the Bohr-Sommerfeldtype quantization condition

$$
\operatorname{Re} \lambda_{\mathrm{SP}}\left(\omega_{\text {lSP }}^{(0)}\right)=\ell \quad \ell=0,1,2, \ldots
$$

Thus, they can be obtained numerically from the dispersion curves. Now, by assuming that $\omega$ is in the neighborhood of $\omega_{\ell \mathrm{SP}}^{(0)}$, we can expand $\lambda_{\mathrm{SP}}(\omega)$ in a Taylor series about $\omega_{\ell S P}^{(0)}$ and write

$$
\begin{aligned}
& \lambda_{\mathrm{SP}}(\omega) \approx \ell+\left.\frac{d \operatorname{Re} \lambda_{\mathrm{SP}}(\omega)}{d \omega}\right|_{\omega=\omega_{\ell \mathrm{SP}}^{(0)}}\left(\omega-\omega_{\ell \mathrm{SP}}^{(0)}\right) \\
& \quad+i \operatorname{Im} \lambda_{\mathrm{SP}}\left(\omega_{\ell \mathrm{SP}}^{(0)}\right)+\left.i \frac{d \operatorname{Im} \lambda_{\mathrm{SP}}(\omega)}{d \omega}\right|_{\omega=\omega_{\ell \mathrm{SP}}^{(0)}}\left(\omega-\omega_{\ell \mathrm{SP}}^{(0)}\right) \\
& \quad+\ldots
\end{aligned}
$$

Then, by replacing (32) in the term $\sin \left[\pi \lambda_{\mathrm{SP}}(\omega)\right]$ of (25), we can see that $f_{\mathrm{SP}}(\omega, \theta)$ presents a resonant behavior given by the Breit-Wigner formula (30) with

$$
\frac{\Gamma_{\ell \mathrm{SP}}}{2}=\left.\frac{\operatorname{Im} \lambda_{\mathrm{SP}}(\omega)\left(d \operatorname{Re} \lambda_{\mathrm{SP}}(\omega) / d \omega\right)}{\left(d \operatorname{Re} \lambda_{\mathrm{SP}}(\omega) / d \omega\right)^{2}+\left(d \operatorname{Im} \lambda_{\mathrm{SP}}(\omega) / d \omega\right)^{2}}\right|_{\omega=\omega_{\ell \mathrm{SP}}^{(0)}} .
$$

Our reasoning also predicts a shift in the excitation frequency $\omega_{\ell S P}^{(0)}$ solution of Eq. (31) which is given by

$$
\Delta \omega_{\ell \mathrm{SP}}^{(0)}=-\left.\frac{\operatorname{Im} \lambda_{\mathrm{SP}}(\omega)\left(d \operatorname{Im} \lambda_{\mathrm{SP}}(\omega) / d \omega\right)}{\left(d \operatorname{Re} \lambda_{\mathrm{SP}}(\omega) / d \omega\right)^{2}+\left(d \operatorname{Im} \lambda_{\mathrm{SP}}(\omega) / d \omega\right)^{2}}\right|_{\omega=\omega_{\ell \mathrm{SP}}^{(0)}} .
$$

In fact, such a shift is always negligible and it can be forgotten. Furthermore, it should be noted that in some frequency range we have

$$
\left|d \operatorname{Re} \lambda_{\mathrm{SP}}(\omega) / d \omega\right| \gg\left|d \operatorname{Im} \lambda_{\mathrm{SP}}(\omega) / d \omega\right|
$$

so that (33) then reduces to

$$
\frac{\Gamma_{\ell \mathrm{SP}}}{2}=\left.\frac{\operatorname{Im} \lambda_{\mathrm{SP}}(\omega)}{d \operatorname{Re} \lambda_{\mathrm{SP}}(\omega) / d \omega}\right|_{\omega=\omega_{\ell \mathrm{SP}}^{(0)}} .
$$

Equations (31) and (33) (or (36) ) are two semiclassical formulas which permit us to determine the location of the resonances from the Regge trajectory of $\lambda_{\mathrm{SP}}$. In Figs. 10 13 we present samples of complex frequencies for the RSPM's associated with the surface waves $\mathrm{SP}_{\infty}$ and $\mathrm{WGSP}_{n}$ with $n=1,2$ and 3. They have been calculated from the semiclassical formulas (31) and (33) by using 
the Regge trajectories determined numerically by solving Eqs. (23) and (24) (see Figs. 69 9). A comparison between the semiclassical spectra and the "exact ones" (calculated by solving numerically Eqs. (15) or (16)) shows a very good agreement. Moreover, we can also observe some interesting features:

- The resonance spectrum associated with the surface wave $\mathrm{SP}_{\infty}$ of the TE theory (see Fig. 10) extends beyond the frequency range $] \omega_{0}, \omega_{b}$ [ where the cylinder presents left-handed behavior because $\mathrm{SP}_{\infty}$ exists for $\omega \in] 0, \omega_{s}[$. Furthermore, inserted into the semiclassical formulas (31) and (33), the behavior of the Regge trajectory of $\lambda_{\mathrm{SP}}$ near $\omega_{s}$ easily explains the existence of the family of resonances close to the real axis of the complex $\omega$ plane which converges for large $\ell$ to the limiting frequency $\omega_{s}$.

- The resonance spectrum associated with the surface wave $\mathrm{SP}_{\infty}$ of the TM theory (see Fig. 12) fully lies inside the frequency range $] \omega_{0}, \omega_{b}[$ where the cylinder presents left-handed behavior because $\mathrm{SP}_{\infty}$ exists only in that range. Furthermore, inserted into the semiclassical formulas (31) and (33), the behavior of the Regge trajectory of $\lambda_{\mathrm{SP}}$ near $\omega_{f}$ explains the existence of the family of resonances close to the real axis of the complex $\omega$ plane which converges for large $\ell$ to the limiting frequency $\omega_{f}$.

- The resonance spectra associated with the surface waves $\mathrm{WGSP}_{n}$ with $n \in \mathbb{N}$ of the TE and TM theories (see Figs. 11 and [13) fully lie inside the frequency range $] \omega_{0}, \omega_{b}$ [ where the cylinder presents left-handed behavior because all these surface waves exist only in that range. Furthermore, inserted into the semiclassical formulas (31) and (33), the behavior of the Regge trajectory of a given Regge pole $\lambda_{\mathrm{WGSP}_{n}}$ near $\omega_{0}$ explains the existence of a corresponding family of resonances close to the real axis of the complex $\omega$ plane which converges for large $\ell$ to the limiting frequency $\omega_{0}$. Since there is an infinity of surface waves $\mathrm{WGSP}_{n}$, the accumulation of resonances in Fig. 10 (resp. Fig. 2b) is more intense at the point $\omega_{0}$ than at the point $\omega_{s}\left(\operatorname{resp} . \omega_{f}\right)$.

In conclusion, we have established a connection between the complex frequencies of the long-lived resonant modes (or RSPM's) of the cylinder and the SP's noted $\mathrm{SP}_{\infty}$ and $\mathrm{WGSP}_{n}$ with $n \in \mathbb{N}$ which are supported by its surface. In other words, in spite of the great confusion which seems to prevail in the resonance spectrum of the left-handed cylinder (see Sec. II), we have been able to fully classify and physically interpret the resonances thanks to CAM techniques. We now invite the reader to look at Figs. 14 and 15] where we have zoomed in on Figs. 17 and 27. On the total cross sections $\sigma_{T}^{H}(\omega)$ and $\sigma_{T}^{E}(\omega)$ we have identified the peaks corresponding to resonances and, for each one, we have specified the SP which has generated it as well as the associated "quantum number" $\ell$.

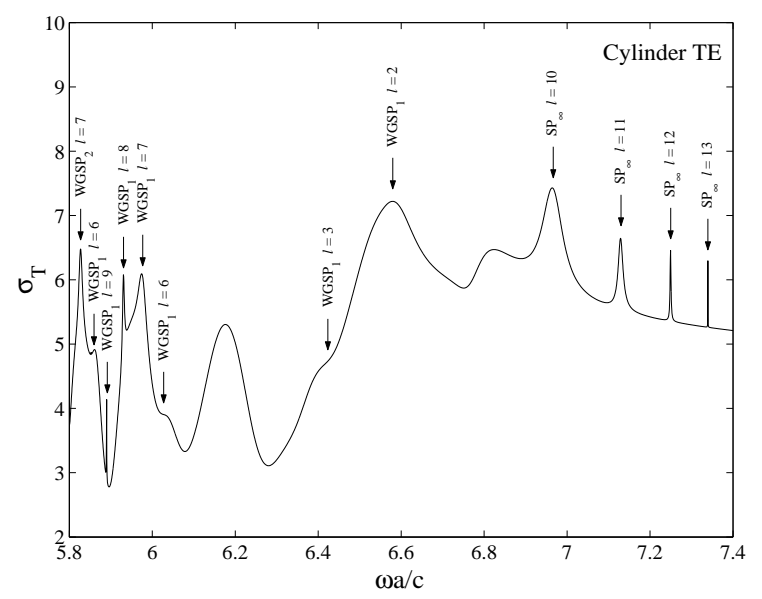

FIG. 14: Zoom-in on the total cross section $\sigma_{T}^{H}$.

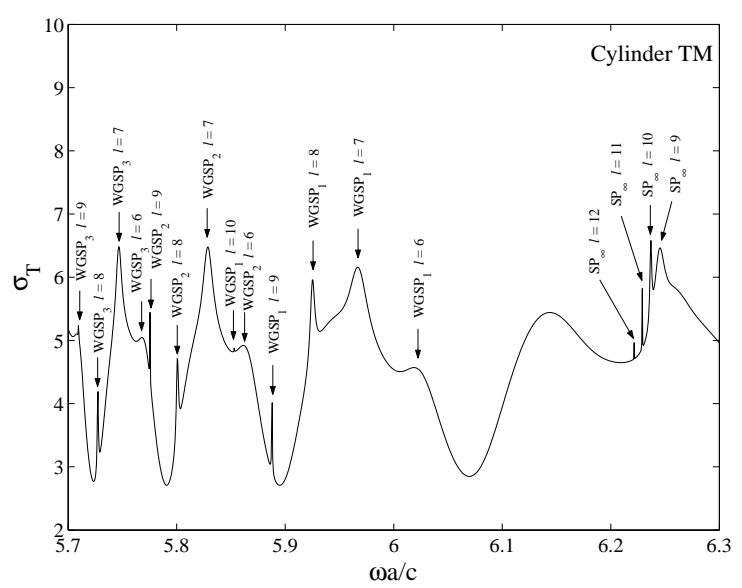

FIG. 15: Zoom-in on the total cross section $\sigma_{T}^{E}$.

\section{ASYMPTOTICS FOR SURFACE POLARITONS AND PHYSICAL DESCRIPTION}

In order to obtain a deeper physical understanding of the SP's orbiting around the left-handed cylinder and to justify the terminology previously used, we must "analytically" solve Eqs. (23) and (24) for $\lambda=\lambda_{\mathrm{SP}}$ or equivalently

$$
-\frac{J_{\lambda_{\mathrm{SP}}}^{\prime}(|n(\omega)| \omega a / c)}{J_{\lambda_{\mathrm{SP}}}(|n(\omega)| \omega a / c)}=\sqrt{\frac{\epsilon(\omega)}{\mu(\omega)}} \frac{H_{\lambda_{\mathrm{SP}}}^{(1)^{\prime}}(\omega a / c)}{H_{\lambda_{\mathrm{SP}}}^{(1)}(\omega a / c)}
$$

for the TE theory and

$$
-\frac{J_{\lambda_{\mathrm{SP}}}^{\prime}(|n(\omega)| \omega a / c)}{J_{\lambda_{\mathrm{SP}}}(|n(\omega)| \omega a / c)}=\sqrt{\frac{\mu(\omega)}{\epsilon(\omega)}} \frac{H_{\lambda_{\mathrm{SP}}}^{(1)^{\prime}}(\omega a / c)}{H_{\lambda_{\mathrm{SP}}}^{(1)}(\omega a / c)}
$$

for the TM theory. Here, Eqs. (37) and (38) have been obtained from Eqs. (23) and (24) by using the relations $J_{\lambda}(-z)=e^{i \pi \lambda} J_{\lambda}(z)$ and $J_{\lambda}^{\prime}(-z)=-e^{i \pi \lambda} J_{\lambda}^{\prime}(z)$ (see Ref. 46). These two equations can be solved approximatively by using asymptotic analysis. More precisely, 
by considering adequate asymptotic expansions for the Bessel functions occurring in (37) and (38), we shall be able to provide analytic expressions for the Regge poles $\lambda_{\mathrm{SP}_{\infty}}(\omega)$ and $\lambda_{\mathrm{WGSP}_{n}}(\omega)$ for the two polarizations. Of course, the main difficulty with this method concerns the choice of the asymptotic expansions for the Bessel functions. Such a choice strongly depends on the relative positions of the arguments $|n(\omega)| \omega a / c$ and $\omega a / c$ with respect to the complex order $\lambda_{\mathrm{SP}}$. In order to simplify the discussion, we choose to describe theoretically the SP's in the frequency ranges where they generate the RSPM's with the longest lifetime (such modes are the most important from the physical point of view). In other words, we shall seek $\lambda_{\mathrm{SP}_{\infty}}(\omega)$ of the TE theory for $\omega$ in the neighborhood of $\omega_{s}, \lambda_{\mathrm{SP}_{\infty}}(\omega)$ of the TM theory for $\omega$ in the neighborhood of $\omega_{f}$ and $\lambda_{\mathrm{WGSP}_{n}}(\omega)$ of the TE and TM theories for $\omega$ in the neighborhood of $\omega_{0}$. In fact, in spite of these restrictions, we shall obtain asymptotic results valid in large frequency ranges.

Let us first consider the Regge pole associated with $\mathrm{SP}_{\infty}$ of the TE theory. We assume $\omega$ in the neighborhood of $\omega_{s}$ and then we can also assume that $\operatorname{Re} \lambda_{\mathrm{SP}_{\infty}}(\omega)>$ $\omega a / c>|n(\omega)| \omega a / c$ and formally that $\operatorname{Re} \lambda_{\mathrm{SP}_{\infty}}(\omega) \gg 1$ and $\operatorname{Re} \lambda_{\mathrm{SP}}(\omega) \gg \operatorname{Im} \lambda_{\mathrm{SP}_{\infty}}(\omega)$. The configuration there is displayed in Fig. 迎. As a consequence, we can use the Debye asymptotic expansions for $J_{\lambda}(z)$ and $H_{\lambda}^{(1)}(z)$ valid for large orders (see Appendix A of Ref. 48 or Ref. 49) in the form

$$
\begin{aligned}
& J_{\lambda}(z) \sim \frac{1}{2} A(\lambda, z) e^{+\alpha(\lambda, z)}, \\
& H_{\lambda}^{(1)}(z) \sim-i A(\lambda, z) e^{-\alpha(\lambda, z)},
\end{aligned}
$$

where

$$
\begin{aligned}
& A(\lambda, z)=\left(\frac{2}{\pi}\right)^{1 / 2}\left(\lambda^{2}-z^{2}\right)^{-1 / 4}, \\
& \alpha(\lambda, z)=\left(\lambda^{2}-z^{2}\right)^{1 / 2}-\lambda \ln \left(\frac{\lambda+\left(\lambda^{2}-z^{2}\right)^{1 / 2}}{z}\right) .
\end{aligned}
$$

Thus, we have

$$
\frac{J_{\lambda_{\mathrm{SP}}}^{\prime}(|n(\omega)| \omega a / c)}{J_{\lambda_{\mathrm{SP}_{\infty}}}(|n(\omega)| \omega a / c)} \sim \frac{\left[\lambda_{\mathrm{SP}_{\infty}}^{2}-(|n(\omega)| \omega a / c)^{2}\right]^{1 / 2}}{|n(\omega)|(\omega a / c)}
$$

and

$$
\frac{H_{\lambda_{\mathrm{SP}_{\infty}}}^{(1)^{\prime}}(\omega a / c)}{H_{\lambda_{\mathrm{SP}_{\infty}}}^{(1)}(\omega a / c)} \sim-\frac{\left[\lambda_{\mathrm{SP}_{\infty}}^{2}-(\omega a / c)^{2}\right]^{1 / 2}}{(\omega a / c)} .
$$

Now, by inserting (42) and (43) into Eq. (37), we obtain an equation which can be easily solved and we deduce

$$
\lambda_{\mathrm{SP}_{\infty}}(\omega) \sim\left(\frac{\omega a}{c}\right) \sqrt{\frac{|\epsilon(\omega)|(|\epsilon(\omega)|+\mu(\omega))}{\epsilon^{2}(\omega)-1}} .
$$

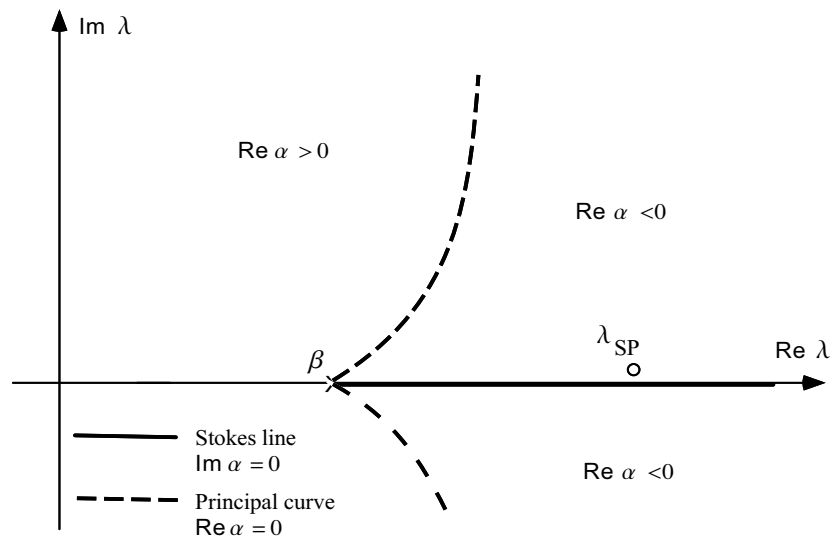

FIG. 16: The relative positions, in the $\lambda$ complex plane, of the reduced frequency $\beta=\omega a / c$ and the Regge pole $\lambda_{\mathrm{SP}_{\infty}}$ of the TE theory.

We have obtained an asymptotic expansion for $\lambda_{\mathrm{SP}_{\infty}}(\omega)$ or, more exactly, for the real part of that function. Indeed, it should be noted that the right-hand side of (44) is purely real. The perturbative method previously used did not permit us to extract the small imaginary part of $\lambda_{\mathrm{SP}_{\infty}}(\omega)$. Even by taking into account higher orders in the asymptotic expansions (39) and (40), we are not able to provide the imaginary part of $\lambda_{\mathrm{SP}_{\infty}}(\omega)$ because it corresponds to an exponentially small contribution which lies beyond all orders in perturbation theory and which can be captured only by carefully taking into account the Stokes phenomenon ${ }^{50,51}$. We have already encountered such a problem in Ref. 40 and, mutatis mutandis, we shall solve it in the same way. Instead of (40), we must use the Debye asymptotic expansion of $H_{\lambda}^{(1)}(z)$ in the form

$$
\begin{aligned}
& H_{\lambda}^{(1)}(z) \sim-i A(\lambda, z) e^{-\alpha(\lambda, z)}(1+\ldots) \\
& \quad+S[\alpha(\lambda, z)] A(\lambda, z)(1+\ldots) e^{\alpha(\lambda, z)} .
\end{aligned}
$$

In the right-hand side of (45), the first term is the usual Debye asymptotic expansion truncated near its least term. The second one is obtained by decoding the divergent tail of that asymptotic expansion. This can be done (see Refs. 42 43) by Borel summation after exploiting a resurgence formula discovered by Dingle ${ }^{41}$. In the region of the $\lambda$ complex plane where the Regge pole $\lambda_{\mathrm{SP}_{\infty}}$ lies (see Fig. [16), we have Re $\alpha<0$. As a consequence, the first term of the right-hand side of (45) is the dominant contribution while the second one is a subdominant term which can be forgotten when $|\lambda| \rightarrow \infty$. That is what we did previously by using (40). The Stokes multiplier function $S[\alpha(\lambda, z)]$ is a complicated function involving the exponential integral function $E_{1}$. It goes continuously from 0 to 1 at the crossing of the Stokes line $\operatorname{Im} \alpha=0$ emerging from the turning point $z=\beta$ (see Fig. 16). Below the Stokes line, it rapidly vanishes. On the Stokes line it is equal to $1 / 2$ and above the Stokes line it rapidly becomes equal to 1 . It thus describes the rapid but con- 
tinuous birth of the subdominant contribution near the Stokes line 42 . From (45) we can now write

$$
\begin{aligned}
& \frac{H_{\lambda_{\mathrm{SP}}}^{(1)^{\prime}}(\omega a / c)}{H_{\lambda_{\mathrm{SP}_{\infty}}}^{(1)}(\omega a / c)} \sim-\frac{\left[\lambda_{\mathrm{SP}_{\infty}}^{2}-(\omega a / c)^{2}\right]^{1 / 2}}{(\omega a / c)} \\
& \quad \times\left(1-2 i S\left[\alpha \left(\lambda_{\left.\left.\left.\mathrm{SP}_{\infty}, \omega a / c\right)\right] e^{2 \alpha\left(\lambda_{\left.\mathrm{SP}_{\infty}, \omega a / c\right)}\right.}\right)}\right.\right.\right.
\end{aligned}
$$

instead of (43). Because we can assume that $\lambda_{\mathrm{SP}_{\infty}}(\omega)$ is very close to the Stokes line emerging from the turning point $\omega a / c$, we can consider that $S\left[\alpha\left(\lambda_{\mathrm{SP}_{\infty}}(\omega), \omega a / c\right)\right]=$ $1 / 2$. Then, by inserting (42) and (46) into Eq. (37), we obtain

$$
\begin{aligned}
\operatorname{Re} \lambda_{\mathrm{SP}_{\infty}}(\omega) & \sim\left(\frac{\omega a}{c}\right) \sqrt{\frac{|\epsilon(\omega)|(|\epsilon(\omega)|+\mu(\omega))}{\epsilon^{2}(\omega)-1}}, \\
\operatorname{Im} \lambda_{\mathrm{SP}_{\infty}}(\omega) & \sim\left(\frac{\epsilon^{2}(\omega)}{\epsilon^{2}(\omega)-1}\right) \\
\times & \frac{\left(\operatorname{Re} \lambda_{\left.\mathrm{SP}_{\infty}(\omega)\right)^{2}-(\omega a / c)^{2}}\right.}{\operatorname{Re} \lambda_{\mathrm{SP}_{\infty}}(\omega)} \\
& \times \exp \left[2 \alpha\left(\operatorname{Re} \lambda_{\mathrm{SP}_{\infty}}(\omega), \omega a / c\right)\right] .
\end{aligned}
$$

Equations (47a) and (47b) provide analytic expressions for the dispersion relation and the damping of the surface polariton $\mathrm{SP}_{\infty}$ of the TE theory. The following important features must be noted:

- The wave number $k_{\mathrm{SP}_{\infty}}(\omega)$ associated with this $\mathrm{SP}$ is obtained from (47a) and (29) and is given by

$$
k_{\mathrm{SP}_{\infty}}(\omega) \sim\left(\frac{\omega}{c}\right) \sqrt{\frac{|\epsilon(\omega)|(|\epsilon(\omega)|+\mu(\omega))}{\epsilon^{2}(\omega)-1}} .
$$

This expression is the usual dispersion relation found in Refs. 363738 for the $p$-polarized SP - i.e., the SP for which the magnetic field $\mathbf{H}$ is normal to the incidence plane - supported by the flat interface. Here, we have recovered exactly the same dispersion relation because we have limited the perturbative resolution of Eq. (37) to the lowest order. By taking into account higher orders in the asymptotic expansions (39) and (45), we could obtain corrections for (48) which vanish for $a \rightarrow \infty-$ i.e., in the flat interface limit. The surface wave of the TE theory denoted by $\mathrm{SP}_{\infty}$ is therefore the counterpart of the $p$-polarized SP supported by the flat interface.

- The imaginary part (47b) of $\lambda_{\mathrm{SP}_{\infty}}$ vanishes for $a \rightarrow \infty$ - i.e., in the flat interface limit. Then, $\mathrm{SP}_{\infty}$ has no damping like the $p$-polarized SP supported by the flat interface. This result strengthens our previous claim.

- The function $\operatorname{Re} \lambda_{\mathrm{SP}_{\infty}}(\omega)$ given by (47a) has a simple pole when $\epsilon(\omega)+1=0$ - i.e., for $\omega=\omega_{s}$. Furthermore, the imaginary part (47b) of $\lambda_{\mathrm{SP}_{\infty}}$ vanishes for $\omega=\omega_{s}$. These two results justify all our previous remarks concerning the accumulation of resonances which converges to the limiting frequency $\omega_{s}$.
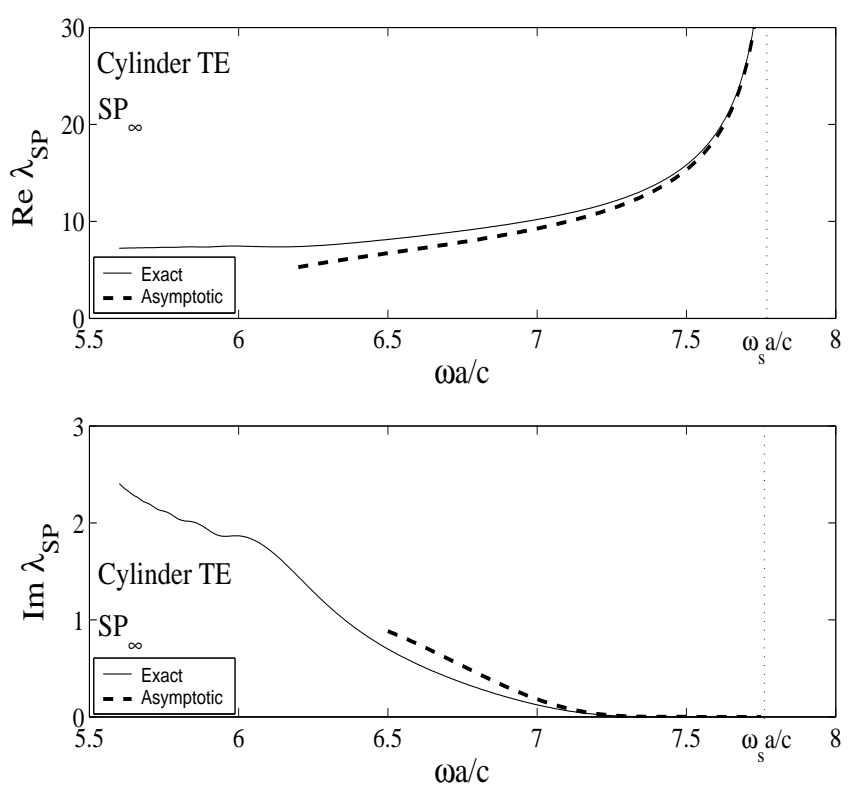

FIG. 17: Regge trajectory for the Regge pole associated with $\mathrm{SP}_{\infty}$ (TE theory). Comparison between exact and asymptotic theories.
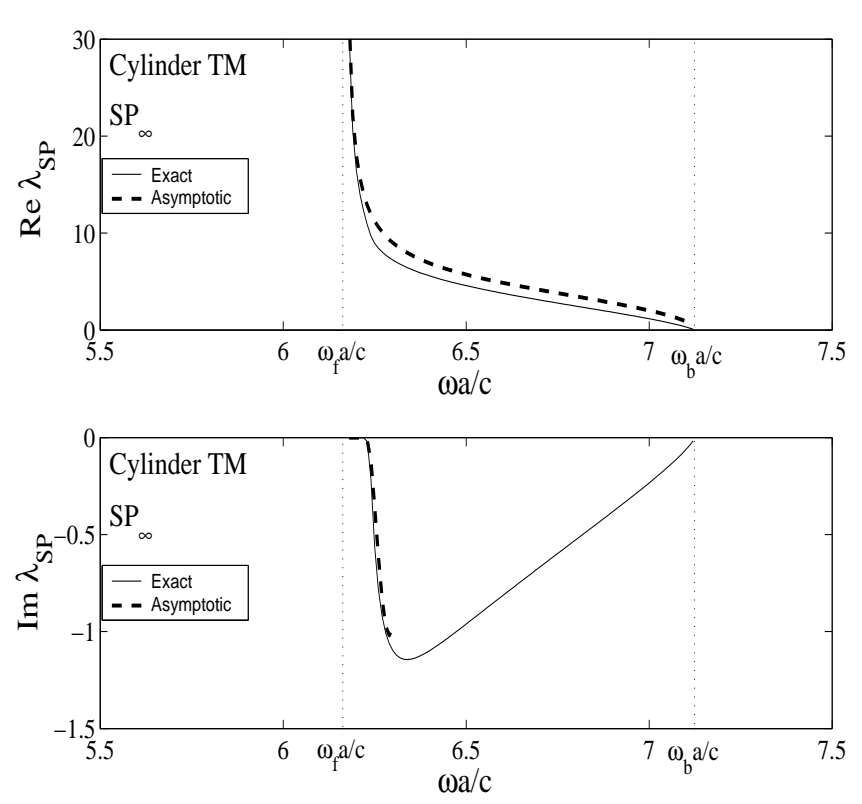

FIG. 18: Regge trajectory for the Regge pole associated with $\mathrm{SP}_{\infty}$ (TM theory). Comparison between exact and asymptotic theories.

- We have numerically tested formulas 47a and (47b) (see Fig. 17). They provide rather good approximations for $\operatorname{Re} \lambda_{\mathrm{SP}_{\infty}}(\omega)$ in a large frequency range and for $\operatorname{Im} \lambda_{\mathrm{SP}_{\infty}}(\omega)$ in the neighborhood of $\omega_{s}$. It would be possible to improve (47b) by taking into account the variation of the Stokes multiplier and to obtain a formula valid in a larger frequency domain but then the expression of $\operatorname{Im} \lambda_{\mathrm{SP}_{\infty}}(\omega)$ would become much more complicated. 
Let us now consider the Regge pole associated with $\mathrm{SP}_{\infty}$ of the TM theory. We must solve Eq. (38) which only differs from Eq. (37) by the factor in front of the Bessel functions. We assume $\omega$ in the neighborhood of $\omega_{f}$ and then we can also assume that $\operatorname{Re} \lambda_{\mathrm{SP}_{\infty}}(\omega)>$ $|n(\omega)| \omega a / c>\omega a / c$ and formally that $\operatorname{Re} \lambda_{\mathrm{SP}_{\infty}}(\omega) \gg 1$ and $\operatorname{Re} \lambda_{\mathrm{SP}_{\infty}}(\omega) \gg \operatorname{Im} \lambda_{\mathrm{SP}_{\infty}}(\omega)$. The configuration there is displayed in Fig. 50. As a consequence, we can use again the Debye asymptotic expansions for $J_{\lambda}(z)$ and $H_{\lambda}^{(1)}(z)$ given in (39) and (45) and the resolution of Eq. (38) can be modelled on that of Eq. (37). Formulas (42) and (46) are still valid and the Stokes multiplier in (46) can be taken equal to $1 / 2$. Indeed, in this case $\lambda_{\mathrm{SP}_{\infty}}(\omega)$ is below the Stokes line emerging from the turning point $\omega a / c$ but it remains very close to this Stokes line. Then, by inserting (42) and (46) into Eq. (38), we obtain

$$
\begin{aligned}
\operatorname{Re} \lambda_{\mathrm{SP}_{\infty}}(\omega) & \sim\left(\frac{\omega a}{c}\right) \sqrt{\frac{|\mu(\omega)|(|\mu(\omega)|+\epsilon(\omega))}{\mu^{2}(\omega)-1}}, \\
\operatorname{Im} \lambda_{\mathrm{SP}_{\infty}}(\omega) & \sim\left(\frac{\mu^{2}(\omega)}{\mu^{2}(\omega)-1}\right) \\
& \times \frac{\left(\operatorname{Re} \lambda_{\mathrm{SP}_{\infty}}(\omega)\right)^{2}-(\omega a / c)^{2}}{\operatorname{Re} \lambda_{\mathrm{SP}_{\infty}}(\omega)} \\
& \times \exp \left[2 \alpha\left(\operatorname{Re} \lambda_{\mathrm{SP}_{\infty}}(\omega), \omega a / c\right)\right] .
\end{aligned}
$$

Equations (49a) and (49b) provide analytic expressions for the dispersion relation and the damping of the surface polariton $\mathrm{SP}_{\infty}$ of the TM theory. The following important features must be also noted:

- The wave number $k_{\mathrm{SP}_{\infty}}(\omega)$ associated with this $\mathrm{SP}$ is obtained from (49a) and (29) and is given by

$$
k_{\mathrm{SP}_{\infty}}(\omega) \sim\left(\frac{\omega}{c}\right) \sqrt{\frac{|\mu(\omega)|(|\mu(\omega)|+\epsilon(\omega))}{\mu^{2}(\omega)-1}} .
$$

This expression is the usual dispersion relation found in Refs. 363738 for the $s$-polarized SP - i.e., the SP for which the electric field $\mathbf{E}$ is normal to the incidence plane - supported by the flat interface. The surface wave of the $\mathrm{TM}$ theory denoted by $\mathrm{SP}_{\infty}$ is therefore the counterpart of the $s$-polarized SP supported by the flat interface.

- The imaginary part (49b) of $\lambda_{\mathrm{SP}_{\infty}}$ vanishes for $a \rightarrow \infty$ - i.e. in the flat interface limit. Then, $\mathrm{SP}_{\infty}$ has no damping like the $s$-polarized SP supported by the flat interface. This result strengthens our previous claim.

- The function $\operatorname{Re} \lambda_{\mathrm{SP}_{\infty}}(\omega)$ given by 49a has a simple pole when $\mu(\omega)+1=0$-i.e., for $\omega=\omega_{f}$. Furthermore, the imaginary part (49b) of $\lambda_{\mathrm{SP}}$ vanishes for $\omega=\omega_{f}$. These two results justify all our previous remarks concerning the accumulation of resonances which converges to the limiting frequency $\omega_{f}$.

- We have numerically tested formulas 49a and (49b) (see Fig. 18). They provide good approximations for $\operatorname{Re} \lambda_{\mathrm{SP}_{\infty}}(\omega)$ in the frequency range $] \omega_{f}, \omega_{b}[$ and for

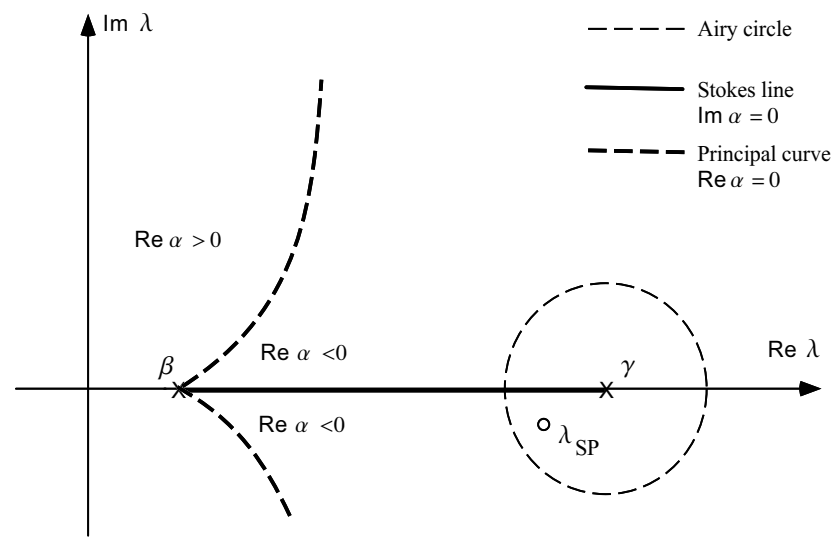

FIG. 19: The relative positions, in the $\lambda$ complex plane, of the reduced frequencies $\beta=\omega a / c$ and $\gamma=|n(\omega)| \omega a / c$ and of the Regge pole $\lambda_{\mathrm{WGSP}_{1}}$ of the TE and TM theories.

$\operatorname{Im} \lambda_{\mathrm{SP}_{\infty}}(\omega)$ in the neighborhood of $\omega_{f}$. Of course, it would be possible to improve (49b) by taking into account the variation of the Stokes multiplier and to obtain a formula for the imaginary part of $\lambda_{\mathrm{SP}_{\infty}}(\omega)$ valid in a larger frequency domain.

Let us finally consider the Regge poles associated with the surface waves $\mathrm{WGSP}_{n}$ for the TE and TM theories. We must now solve Eq. (37) and Eq. (38) for $\lambda_{\mathrm{SP}}=\lambda_{\mathrm{WGSP}_{n}}$ by assuming $\omega$ in the neighborhood of $\omega_{0}$. We are in the configurations described in Fig. 4 a for the TE theory and Fig. 5 a for the TM theory (see also Fig. 19). As far as the asymptotic expansion for $H_{\lambda}(z)$ is concerned, we can still use (40) as well as its modification (45) because we can assume that $\operatorname{Re} \lambda_{\mathrm{WGSP}_{n}}(\omega)>\omega a / c$ and formally that $\operatorname{Re} \lambda_{\mathrm{WGSP}_{n}}(\omega) \gg 1$ and $\operatorname{Re} \lambda_{\mathrm{WGSP}_{n}}(\omega) \gg \operatorname{Im}_{\mathrm{WGSP}_{n}}(\omega)$. We then obtain

$$
\begin{aligned}
& \frac{H_{\lambda_{\mathrm{WGSP}_{n}}^{(1)^{\prime}}}^{(1)}(\omega a / c)}{H_{\lambda_{\mathrm{WGSP}_{n}}}^{(1)}(\omega a / c)} \sim-\frac{\left[\lambda_{\mathrm{WGSP}_{n}}^{2}-(\omega a / c)^{2}\right]^{1 / 2}}{(\omega a / c)} \\
& \times\left(1-2 i S\left[\alpha\left(\lambda_{\mathrm{WGSP}_{n}}, \omega a / c\right)\right] e^{2 \alpha\left(\lambda_{\mathrm{WGSP}_{n}}, \omega a / c\right)}\right) .
\end{aligned}
$$

As far as the asymptotic expansion for $J_{\lambda}(z)$ is concerned, the situation is a little bit more complicated. $\lambda_{\mathrm{WGSP}_{n}}$ is in the immediate neighborhood of $|n(\omega)| \omega a / c$ or more precisely in the Airy circle centered on $|n(\omega)| \omega a / c$ (see Fig. 19). This is true for the Regge pole corresponding to $n=1$ but it is also formally true for all the other Regge poles with $n \neq 1$ when $\omega \rightarrow \omega_{0}$. Then, the Debye asymptotic expansion (40) for $J_{\lambda}(z)$ fails because $\lambda \approx z$ and we must use the uniform asymptotic expansion (see Appendix A of Ref. 48 or Ref. 49)

$$
J_{\lambda}(z) \sim(2 / z)^{1 / 3} A i\left[(2 / z)^{1 / 3}(\lambda-z)\right]
$$


where $A i(z)$ denotes the Airy function ${ }^{46}$. Thus we have

$$
\begin{aligned}
& \frac{J_{\lambda_{\mathrm{WGSP}_{n}}^{\prime}}^{\prime}(|n(\omega)| \omega a / c)}{J_{\lambda_{\mathrm{WGSP}_{n}}}(|n(\omega)| \omega a / c)} \sim(2 /(|n(\omega)| \omega a / c))^{1 / 3} \\
& \times \frac{A i^{\prime}\left[(2 /(|n(\omega)| \omega a / c))^{1 / 3}\left(\lambda_{\mathrm{WGSP}_{n}}-|n(\omega)| \omega a / c\right)\right]}{A i\left[(2 /(|n(\omega)| \omega a / c))^{1 / 3}\left(\lambda_{\mathrm{WGSP}_{n}}-|n(\omega)| \omega a / c\right)\right]}
\end{aligned}
$$

We can now insert (53) and (51) into Eqs. (37) and (38). In order to solve the resulting equations, we first note that their rhs tend to infinity when $\omega \rightarrow \omega_{0}$. Hence, the argument of the Airy function is very close to the zeros $x_{n}$ of that function (let us recall here that the first three ones are $x_{1} \approx-2.3381 \ldots, x_{2} \approx-4.0879 \ldots$ and $x_{3} \approx$ $-5.5205 \ldots)$ and we then have

$$
\lambda_{\mathrm{WGSP}_{n}}(\omega) \sim|n(\omega)| \omega a / c+\left(\frac{|n(\omega)| \omega a / c}{2}\right)^{1 / 3} x_{n} .
$$

Of course, Eq. (54) provides an approximation which can be easily improved by taking into account the subdominant contribution in (511). We assume that the Stokes multiplier is equal to $1 / 2$. This is true because the Regge pole $\lambda_{\mathrm{WGSP}_{n}}(\omega)$ is below but very close to the Stokes line emerging from the turning point $\omega a / c$. We then obtain

$$
\operatorname{Re}_{\mathrm{WGSP}_{n}}(\omega) \sim|n(\omega)| \omega a / c+\left(\frac{|n(\omega)| \omega a / c}{2}\right)^{1 / 3} x_{n}
$$

for the TE and TM theories,

$$
\begin{aligned}
& \operatorname{Im} \lambda_{\mathrm{WGSP}_{n}}(\omega) \sim \sqrt{\frac{\epsilon(\omega)}{\mu(\omega)}}\left(\frac{|n(\omega)| \omega a / c}{2}\right)^{2 / 3} \\
& \times \frac{\left[\left(\operatorname{Re} \lambda_{\operatorname{WGSP}_{n}}(\omega)\right)^{2}-(\omega a / c)^{2}\right]^{1 / 2}}{(\omega a / c) x_{n}} \\
& \times \exp \left[2 \alpha\left(\operatorname{Re} \lambda_{\mathrm{WGSP}_{n}}(\omega), \omega a / c\right)\right]
\end{aligned}
$$

for the TE theory and

$$
\begin{aligned}
\operatorname{Im} \lambda_{\mathrm{WGSP}_{n}}(\omega) \sim \sqrt{\frac{\mu(\omega)}{\epsilon(\omega)}}\left(\frac{|n(\omega)| \omega a / c}{2}\right)^{2 / 3} \\
\times \frac{\left[\left(\operatorname{Re} \lambda_{\operatorname{WGSP}_{n}}(\omega)\right)^{2}-(\omega a / c)^{2}\right]^{1 / 2}}{(\omega a / c) x_{n}} \\
\times \exp \left[2 \alpha\left(\operatorname{Re} \lambda_{\mathrm{WGSP}_{n}}(\omega), \omega a / c\right)\right]
\end{aligned}
$$

for the TM theory .

Equations (55a)-(55d) provide analytic expressions for the dispersion relation and the damping of the surface polaritons $\mathrm{WGSP}_{n}$ of the TE and TM theories. The corresponding wave numbers $k_{\mathrm{WGSP}_{n}}(\omega)$ which are obtained from (55a) and (29) are given by

$$
k_{\mathrm{WGSP}_{n}}(\omega) \sim|n(\omega)| \omega / c+\left(\frac{|n(\omega)| \omega / c}{2 a^{2}}\right)^{1 / 3} x_{n} .
$$
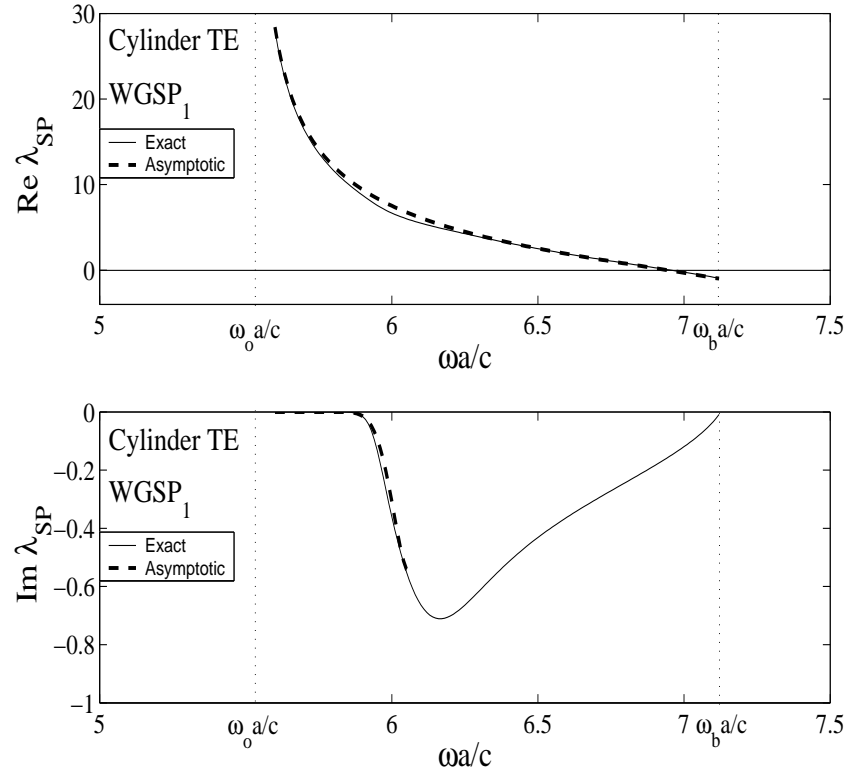

FIG. 20: Regge trajectory for the Regge pole associated with WGSP $_{1}$ (TE theory). Comparison between exact and asymptotic theories.
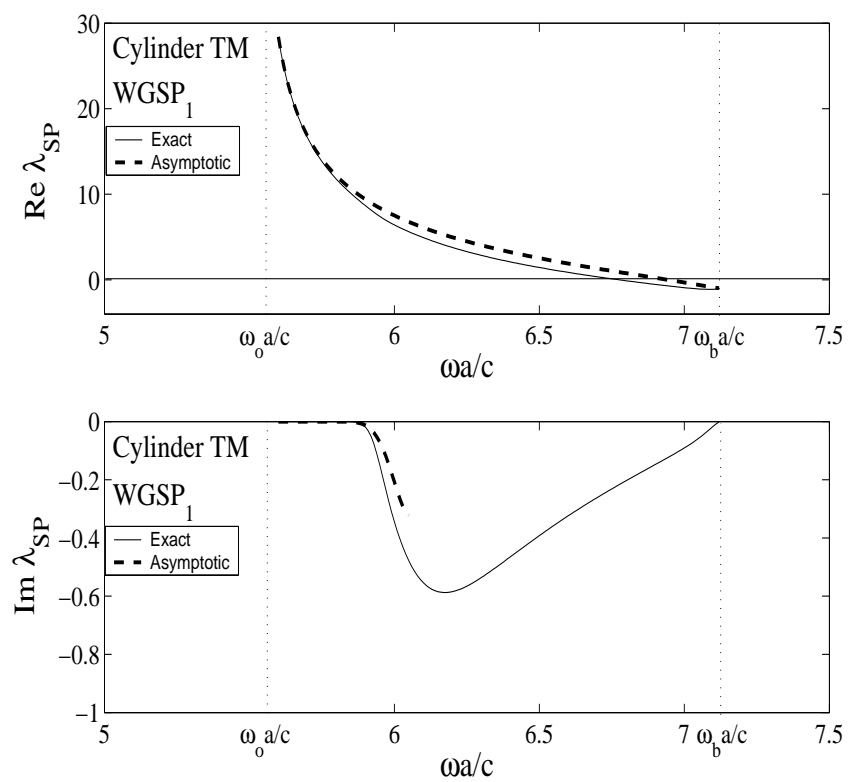

FIG. 21: Regge trajectory for the Regge pole associated with WGSP $_{1}$ (TE theory). Comparison between exact and asymptotic theories.

It should be noted that (54) and therefore (55a) have been obtained by following, mutatis mutandis, the calculations done by Rayleigh a long time ago in order to describe mathematically the whispering-gallery phenomenon in acoustics ${ }^{52.53}$ (see also Ref. 54). We encounter the same phenomenon but in the context of lefthanded electromagnetism and the SP's described by the Regge poles $\lambda_{\mathrm{WGSP}_{n}}$ are surface waves of whispering- 
gallery type. Here it should be recalled that a whisperinggallery surface wave propagates only close to a curved interface and mainly in the internal medium in a layer whose thickness is proportional to $k^{-2 / 3}$ with $k$ its wave number. From a physical point of view, the surface polaritons $\mathrm{WGSP}_{n}$ are therefore very different from the surface polaritons $S P_{\infty}$ which exist on both sides of the interface. Moreover, the following important features must be also noted:

- These SP's have no counterparts in the plane interface case.

- The function $\operatorname{Re} \lambda_{\mathrm{WGSP}_{n}}(\omega)$ given by (49a) has a pole which is that of $n(\omega)$ and therefore which corresponds to $\omega=\omega_{0}$. Furthermore, the imaginary parts (55b) and (55c) of $\lambda_{\mathrm{WGSP}_{n}}$ vanish for $\omega=\omega_{0}$. These results justify all our previous remarks concerning the accumulations of resonances which converge to the limiting frequency $\omega_{0}$.

- We have numerically tested formulas (55a)-(55C) (see Figs. 20] and 21] for WGSP $_{1}$ ). They provide very good approximations for $\operatorname{Re} \lambda_{\mathrm{WGSP}_{1}}(\omega)$ in the full frequency range $] \omega_{0}, \omega_{b}[$ where the cylinder presents left-handed behavior. They also provide very good approximations for $\operatorname{Im} \lambda_{\mathrm{WGSP}_{1}}(\omega)$ in a rather large frequency range above the limiting frequency $\omega_{0}$.

\section{CONCLUSION AND PERSPECTIVES}

In the present article, we have considered the scattering of TE and TM electromagnetic waves by a cylinder fabricated from a left-handed material. We have mainly emphasized the resonant aspects of the problem. We have shown that the long-lived resonant modes can be classified into distinct families, each family being generated by one SP and we have physically described all the SP's orbiting around the cylinder by providing, for each one, a numerical and a semiclassical description of its dispersion relation and its damping.

We have also shown that the left-handed cylindrical interface can support both TE- and TM-polarized SP's. For each polarization, we have found that there exists a particular SP which corresponds, in the large-radius limit, to the SP which is supported by the plane interface and which has been theoretically described in Refs. 36 37 38. But there also exists, for each polarization, an infinite family of SP's of whispering-gallery type and these have no analogs in the plane interface case. The existence of these SP's of whispering-gallery type is certainly the main result of our article. Here, in order to appreciate it, it should be recalled that on a cylindrical metal-dielectric or semiconductor-dielectric interface, the SP's of whispering-gallery type are absent. There exists only one $\mathrm{SP}^{40}$. It can be excited in the $H$ polarization configuration - i.e., by TE waves - and in the large-radius limit it corresponds to the SP which is supported by the plane interface. From the point of view of SP physics, left-handed cylinders are therefore much richer systems than metallic or semiconducting cylinders and this could have important consequences in term of practical applications.

We are now completing a theoretical and numerical study in order to provide a better physical understanding of the resonant modes of left-handed cylinder ${ }^{55}$. We more particularly focus on the repartition of the electromagnetic energy density - both inside and outside the cylinder - associated with all the resonant modes. That enables us to physically distinguish on the one hand the resonant modes generated by bulk polaritons from those generated by SP's, and on the other hand, the resonant modes generated by SP's of $\mathrm{SP}_{\infty}$ type from those generated by SP's of whispering-gallery type.

It should be also noted that in our paper we have assumed for the effective electric permittivity and the effective magnetic permeability of the left-handed material the simple expressions respectively given by (3) and (4). In fact, actual left-handed materials have a more complicated permittivity. Indeed, they consist of periodic arrangements of split ring resonators and wires and it has been recently shown that the split ring resonators present a strong electric response in addition to the magnetic one (see Ref. 56). In fact, it is possible to take into account this response by assuming that the effective electric permittivity is given by ${ }^{56}$

$$
\epsilon(\omega)=\frac{\omega^{2}-\omega_{p}^{2}}{\omega^{2}-\tilde{\omega}_{0}^{2}}
$$

with $\tilde{\omega}_{0} \neq 0$ while the effective magnetic permeability is still given by (4). Such a modification does not change radically our previous analysis. Mutatis mutandis, our results remain valid. Indeed, we have theoretically - i.e., semiclassically - and numerically reexamined, in this new context, the resonant aspects of a left-handed cylinder linked with SP's. We have obtained the following results:

- The surface wave $\mathrm{SP}_{\infty}$ of the TE theory still exists. It generates a family of resonances which converges to the limiting frequency $\omega_{s}$ satisfying $\epsilon\left(\omega_{s}\right)+1=0$ and which is now given by $\omega_{s}=\sqrt{\left(\omega_{p}^{2}+\tilde{\omega}_{0}^{2}\right) / 2}$ instead of (18). This SP is still described by Eqs. (47a) and (47b) with now $\epsilon(\omega)$ given by (57). Of course, its behavior depends on the relative order of the characteristic frequencies $\tilde{\omega}_{0}, \omega_{0}, \omega_{b}, \omega_{p}$. In general, it presents a lefthanded behavior if $\omega_{s}$ lies in the frequency range where $n(\omega)<0$.

- The surface wave $\mathrm{SP}_{\infty}$ of the TM theory still exists. It generates a family of resonances which converges to the limiting frequency $\omega_{f}$ satisfying $\mu\left(\omega_{f}\right)+1=0$ and which is still given by (20). This SP is still described by Eqs. (49a) and (49b) and, in general, it presents a lefthanded behavior if $\omega_{f}$ lies in the frequency range where $n(\omega)<0$.

- For both polarizations, there still exists the family of SP's of whispering-gallery type described by Eqs. (55a)-(55c) in the neighborhood of the pole $\omega_{0}$ of $\mu(\omega)$. They generate an infinity of resonances which converges to the limiting frequency $\omega_{0}$. In general, they 
present a left-handed behavior if $\omega_{0}$ lies in the frequency range where $n(\omega)<0$.

- But there also exists, for both polarizations, a new family of SP's of whispering-gallery type in the neighborhood of the pole $\tilde{\omega}_{0}$ of $\epsilon(\omega)$. They are also described by (55a)-(55c) (in this frequency range) and they generate an infinity of resonances which converges to the limiting frequency $\tilde{\omega}_{0}$. In general, they present a lefthanded behavior if $\tilde{\omega}_{0}$ lies in the frequency range where $n(\omega)<0$.

Finally, it is worthwhile pointing out the possible application of the CAM method in the context of multiple scattering by left-handed cylinders. This could be achieved by extending to electromagnetism of dispersive media the approaches previously developed in the context of quantum mechanics and acoustics (see, for example, Ref. 57 58). SP's probably induce a strong coupling between the different cylinders and the CAM method could permit us to understand it and to quantify its intensity.

\section{Acknowledgments}

We are grateful to Bruce Jensen for help with the English.
* Electronic address: ancey@univ-corse.fr

† Electronic address: decanini@univ-corse.fr

$\ddagger$ Electronic address: folacci@univ-corse.fr

$\S$ Electronic address: gabrieli@univ-corse.fr

1 V. G. Veselago, Sov. Phys. Usp. 10, 509 (1968).

2 D. R. Smith and N. Kroll, Phys. Rev. Lett. 85, 2933 (2000).

3 J. B. Pendry, A. J. Holden, W. J. Stewart, and I. Youngs, Phys. Rev. Lett. 76, 4773 (1996).

4 J. B. Pendry, A. J. Holden, D. J. Robbins, and W. J. Stewart, J. Phys.: Condens. Matter 10, 4785 (1998).

5 J. B. Pendry, A. J. Holden, D. J. Robbins, and W. J. Stewart, IEEE Trans. Microwave Theory Tech. 47, 2075 (1999).

${ }^{6}$ D. R. Smith, W. J. Padilla, D. C. Vier, S. C. Nemat-Nasser, and S. Schultz, Phys. Rev. Lett. 84, 4184 (2000).

7 R. A. Shelby, D. R. Smith, S. C. Nemat-Nasser, and S. Schultz, Appl. Phys. Lett. 78, 4 (2001).

${ }^{8}$ R. A. Shelby, D. R. Smith, and S. Schultz, Science 292, 77 (2001).

9 J. B. Pendry and D. R. Smith, Phys. Today 57, 37 (2004).

10 A. Berrier, M. Mulot, M. Swillo, M. Qiu, L. Thylen, A. Talneau, and S. Anand, Phys. Rev. Lett. 93, 073902 (2004).

11 V. Kuzmiak and A. A. Maradudin, Phys. Rev. B 66, 045116 (2002).

12 R. G. Newton, Scattering Theory of Waves and Particles (Springer-Verlag, New York, 1982), 2nd ed.

13 H. M. Nussenzveig, Diffraction Effects in Semiclassical Scattering (Cambridge University Press, Cambridge, 1992).

14 D. L. Mills and E. Burstein, Rep. Prog. Phys. 37, 817 (1974).

15 V. M. Agranovich and D. L. Mills, Surface Polaritons: Electromagnetic Waves at Surfaces and Intersurfaces (North-Holland, Amsterdam, 1982).

16 H. Raether, Surface Plasmons Vol. 111 of Springer Tracts in Modern Physics (Springer-Verlag, Berlin, 1988).

17 B. E. Sernelius, Surface Modes in Physics (Wiley-VCH Verlag GmbH, Weinheim, 2001).

18 A. R. McGurn and A. A. Maradudin, Phys. Rev. B 48, 17576 (1993).

19 V. Kuzmiak, A. A. Maradudin, and F. Pincemin, Phys. Rev. B 50, 16835 (1994).

${ }^{20}$ M. M. Sigalas, C. T. Chan, K. M. Ho, and C. M. Soukoulis, Phys. Rev. B 52, 11744 (1995).

21 W. Zhang, A. Hu, X. Lei, N. Xu, and N. Ming, Phys. Rev. B 54, 10280 (1996).
${ }^{22}$ V. Kuzmiak, A. A. Maradudin, and A. R. McGurn, Phys. Rev. B 55, 4298 (1997).

${ }^{23}$ V. Kuzmiak and A. A. Maradudin, Phys. Rev. B 55, 7427 (1997).

24 H. van der Lem and A. Moroz, J. Opt. A: Pure Appl. Opt. 2, 395 (2000).

${ }^{25}$ K. Sakoda, Optical Properties of Photonic Crystals (Springer-Verlag, Berlin, 2001).

26 T. Ito and K. Sakoda, Phys. Rev. B 64, 045117 (2001).

27 E. Moreno, D. Erni, and C. Hafner, Phys. Rev. B 65, 155120 (2002).

28 T. Ochiai and J. Sanchez-Dehesa, Phys. Rev. B 65, 245111 (2002).

29 T. W. Ebbesen, H. J. Lezec, H. F. Ghaemi, T. Thio, and P. A. Wolff, Nature (London) 391, 667 (1998).

30 W. L. Barnes, W. A. Murray, J. Dintinger, E. Devaux, and T. W. Ebbesen, Phys. Rev. Lett. 92, 107401 (2004).

31 J. B. Pendry, Phys. Rev. Lett. 85, 3966 (2000).

32 M. W. Feise, P. J. Bevelacqua, and J. B. Schneider, Phys. Rev. B 66, 035113 (2001).

33 F. D. M. Haldane, cond-mat/0206420 (unpublished).

34 X. S. Rao and C. K. Ong, Phys. Rev. B 68, 113103 (2003).

35 I. V. Shadrivov, A. A. Zharov, and Y. S. Kivshar, Appl. Phys. Lett. 83, 2713 (2003).

36 R. Ruppin, Phys. Lett. A 277, 61 (2000).

37 S. A. Darmanyan, M. Nevière, and A. A. Zakhidov, Opt. Commun. 225, 233 (2003).

38 I. V. Shadrivov, A. A. Sukhorukov, Y. S. Kivshar, A. A. Zharov, A. D. Boardman, and P. Egan, Phys. Rev. E 69, 016617 (2004).

39 R. Ruppin, Solid State Comm. 116, 411 (2000).

40 S. Ancey, Y. Décanini, A. Folacci, and P. Gabrielli, Phys. Rev. B 70, 245406 (2004).

41 R. D. Dingle, Asymptotic Expansions: Their Derivation and Interpretation (Academic Press, London, 1973).

42 M. V. Berry, Proc. R. Soc. London A 422, 7 (1989).

43 M. V. Berry and C. J. Howls, Proc. R. Soc. London A 430, 653 (1990).

44 H. Segur, S. Tanveer, and H. Levine, Asymptotics Beyond all Orders (Plenum, New York, 1991).

45 N. F. Mott and H. S. W. Massey, The Theory of Atomic Collisions (Oxford University Press, Oxford, 1965).

46 M. Abramowitz and I. A. Stegun, Handbook of Mathematical Functions (Dover, New-York, 1965).

47 G. N. Watson, Proc. Roy. Soc. London A 100, 83 (1918). 
48 H. M. Nussenzveig, Ann. Phys. (N.Y.) 34, 23 (1965).

49 G. N. Watson, Theory of Bessel Functions (Cambridge University Press, Cambridge, 1995), 2nd ed.

${ }^{50}$ G. G. Stokes, Trans. Cambridge Philos. Soc. 9, 379 (1847).

51 G. G. Stokes, Trans. Cambridge Philos. Soc. 10, 106 (1864).

52 J. W. S. Rayleigh, The Theory of Sound reprinted by Dover (Dover, New York, 1976).

53 J. W. S. Rayleigh, Phil. Mag. 20, 1001 (1910).

${ }^{54}$ W. Streifer and R. D. Kodis, Q. Appl. Math. 21, 285
(1964).

55 S. Ancey, Y. Décanini, A. Folacci, and P. Gabrielli (In preparation).

56 T. Koschny, M. Kafesaki, E. N. Economou, and C. M. Soukoulis, Phys. Rev. Lett. 93, 107402 (2004).

57 A. Wirzba, Phys. Rep. 309, 1 (1999).

58 P. Gabrielli and M. Mercier-Finidori, Phys. Rev. E 66, 046629 (2002). 